\title{
A Hybrid Forecasting Model Based on Empirical Mode Decomposition and the Cuckoo Search Algorithm: A Case Study for Power Load
}

\author{
Jiani Heng, ${ }^{1,2}$ Chen Wang, ${ }^{2}$ Xuejing Zhao, ${ }^{2}$ and Jianzhou Wang ${ }^{1}$ \\ ${ }^{1}$ School of Statistics, Dongbei University of Finance and Economics, Dalian, Liaoning 116025, China \\ ${ }^{2}$ School of Mathematics and Statistics, Lanzhou University, Lanzhou, Gansu 730000, China \\ Correspondence should be addressed to Chen Wang; chenwang15@lzu.edu.cn
}

Received 20 August 2015; Revised 8 April 2016; Accepted 13 April 2016

Academic Editor: Dongsuk Kum

Copyright (C) 2016 Jiani Heng et al. This is an open access article distributed under the Creative Commons Attribution License, which permits unrestricted use, distribution, and reproduction in any medium, provided the original work is properly cited.

Power load forecasting always plays a considerable role in the management of a power system, as accurate forecasting provides a guarantee for the daily operation of the power grid. It has been widely demonstrated in forecasting that hybrid forecasts can improve forecast performance compared with individual forecasts. In this paper, a hybrid forecasting approach, comprising Empirical Mode Decomposition, CSA (Cuckoo Search Algorithm), and WNN (Wavelet Neural Network), is proposed. This approach constructs a more valid forecasting structure and more stable results than traditional ANN (Artificial Neural Network) models such as BPNN (Back Propagation Neural Network), GABPNN (Back Propagation Neural Network Optimized by Genetic Algorithm), and WNN. To evaluate the forecasting performance of the proposed model, a half-hourly power load in New South Wales of Australia is used as a case study in this paper. The experimental results demonstrate that the proposed hybrid model is not only simple but also able to satisfactorily approximate the actual power load and can be an effective tool in planning and dispatch for smart grids.

\section{Introduction}

In a power system, the short-term power load forecasting is very important for the stable operation of the system. Accurate forecasting is a guarantee in the development of preventive maintenance plans, which include generator safeguards, power system reliability estimation, and scheduling dispatch $[1,2]$. High-accuracy power load forecasts improve the economic and social benefits of power grid management, which reduce generation costs, improve the security of power systems, and help administrators develop optimal plans. Moreover, accurate load forecasting is crucial in forecasts of the power price in power markets [3]. Therefore, developing power load forecasting techniques to achieve accurate, simple, and fast load forecasts is necessary. Thus far, many shortterm power load forecasting methods have been proposed, and these methods can be mainly divided into three categories: conventional methods, modern forecasting methods, and hybrid forecasting methods. Conventional methods include multiple linear regression analysis $[4,5]$, time series
$[6,7]$, state space models [8], general exponential smoothing [9], and knowledge-based methods. However, these methods cannot provide appropriate nonlinear mathematical relationships to express actual power loads. The primary modern forecasting methods are intelligent evolutionary algorithms $[10,11]$, expert systems [12, 13], neural networks [14-17], and fuzzy inference [18]. Intelligent algorithms and neural networks obtain good performance because of their clear patterns, easy implementation, and strong ability to address the problem. Hybrid forecasting methods, proposed to avoid the shortcomings that exist in individual forecasting methods, have become increasingly prevalent $[19,20]$. A detailed introduction of the three categories is given below.

The deduction processes of traditional forecasting methods are rigorous, and most of them are based on traditional mathematics theories such as statistics, calculus, and modeling by subjective data analysis [21]. The main idea of trend extrapolation technology is to look for the trend of data changes, according to the trend equation, to forecast future data. The method is simple, and, especially for smooth power 
load changes, it can achieve a good prediction effect. Its deficiency is that its precision is greatly influenced by the random load component [22]. The regression analysis method is often applied to short-term load forecasting [23]. This method has many advantages such as a simple principle and better quality of data which leads to better precision; however, the selection of the main factors affecting power load in the model is difficult as many factors that affect the forecasting accuracy are hard to quantify. This model is lacking any self-study capability, and the input variable and output variable cannot be revised automatically [24]. With years of development, the time series forecasting method has become a mature theory method and has been applied to power load forecasting [25]. The basic time series prediction models mainly include AR, MA, and ARMA [26]. Although the time series forecasting method has advantages such as only requiring a small volume of historical data and a small amount of calculation and the fast speed of its calculation, this method has certain limitations such as its inability to reflect the influence of meteorological factors and how its forecasting accuracy will decrease with the increase of the prediction step [27]. ANN [17] is a type of nonlinear simulation of the human brain information processing system with an intelligent processing process; for an inaccurate variation trend, this method also has a good ability to adapt, is able to grasp information and keep on learning, and has good knowledge reasoning and self-optimization [28]. An expert system is a computer system based on the knowledge of the programming approach, mainly a software system, and the main components of an expert system include the inference engine of the system, the expert knowledge base, the explain interface, and the knowledge acquisition module. An expert system is a program that has decisionmaking capabilities based on reasoned knowledge; however, this method is limited by whether the expert knowledge is complete [29]. The grey forecasting method is an important technique in grey theory, and it uses approximate differential equations to describe future tendencies for a time series [30]. The limitation of this method is that the greater the dispersion degree of data, the worse the forecasting accuracy. Although traditional forecasting methods and forecasting methods based on intelligent computing have their respective applications, it is difficult to achieve better results when using one of them by itself [31]. In the literature related to forecasting [31-34], the forecasting results are not quite as good with any single forecasting model. The primary reason is that single forecasting models cannot extract the complicated factors encountered in reality.

Due to the limitations of the forecasting capacity of a single model, it cannot always be optimal in all cases. In this paper, a novel hybrid model was developed with the hope of obtaining more accurate power load forecasting results. The proposed hybrid wind speed forecasting model can be grouped into four steps. Firstly, we used the empirical model decomposition technique, which represents a nonstationary data analysis technique, to reconstruct the original wind speed series. Secondly, a WNN model was employed to create the power load forecasting, and the parameters in the WNN model were tuned by the CSA. The simulation results illustrate that the hybrid model is an effective method in power load forecasting. The main contributions of this paper are summarized as follows:

(1) The CSA algorithm is applied to choose the optimal initial weight in the WNN model, which always leads to unstable forecasting error.

(2) In the field of power load forecasting, the proposed hybrid model is manifested as a valid method with efficient computation and satisfactory forecasting accuracy.

(3) Considering the skewness and kurtosis of the forecasting accuracy distribution, the developed forecasting availability is proposed as an effective evaluation criterion for model selection in the power load forecasting field.

This paper is organized as follows. First, we outline the concept of models used in this paper, including empirical model decomposition, WNN, CSA, BPNN, GABPNN, and EMD-CSAWNN. Second, the modeling processes of the methods mentioned above are introduced. Simulation results are presented and analyzed. Finally, the overall conclusion is included.

\section{Methodology}

In this section, the required individual tools will be presented concisely, including the empirical model decomposition technique, BPNN, the WNN model, and the CSA and GA algorithms. Moreover, the proposed hybrid approach will be described in detail. In addition, the structure of the feedforward neural network will be confirmed.

2.1. Empirical Mode Decomposition. Empirical model decomposition was proposed by Karthikeyan and Kumar as an adaptive method for nonstationary time series analysis, and it is now widely used. It can be applied to any type of signal decomposition [35]. Thus, it has obvious advantages in processing nonstationary and nonlinear series. The foundation of this technique is to decompose a time series into a finite set of several IMFs and a residue [36].

Definition 1. All IMFs are defined to satisfy the following conditions: (1) the number of local extreme points and the number of zero crossings must be equal or at least differ by only one; (2) the mean value of the upper envelope and lower envelope is zero.

Definition 2. The stoppage criterion determined is defined as

$$
\mathrm{SD}_{k}=\frac{\sum_{t=0}^{T}\left|h_{1 k-1}(t)-h_{1 k}(t)\right|^{2}}{\sum_{t=0}^{T} h_{1 k-1}^{2}(t)}
$$

The sifting process stops when $\mathrm{SD}_{k}$ is smaller than a pregiven value. The process of decomposition is over when the value of $\mathrm{SD}_{k}$ is between 0.2 and 0.3 . Additional details of the 


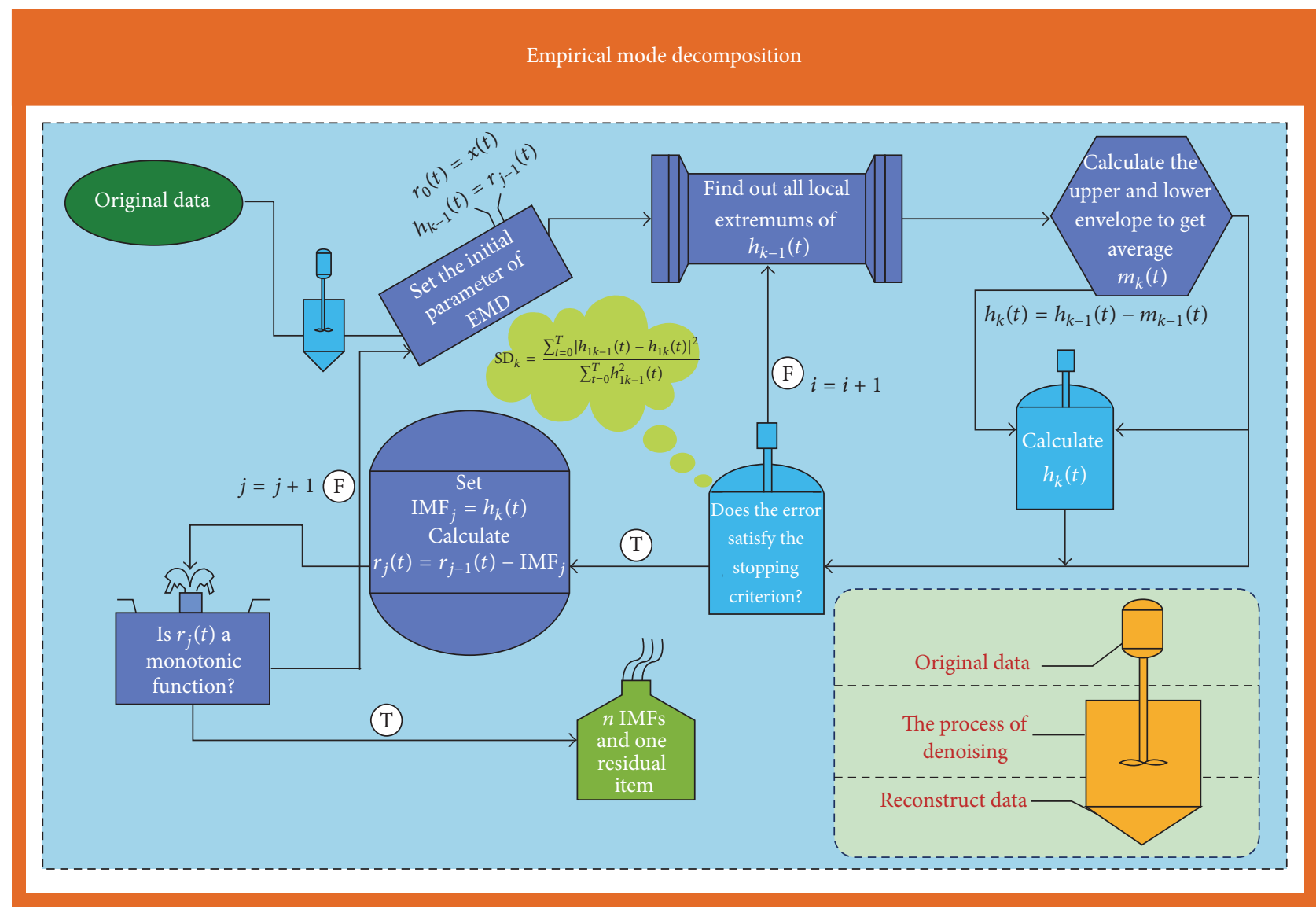

FIGURE 1: The construction of empirical model decomposition.

empirical model decomposition technique are illustrated in Figure 1.

\subsection{Artificial Neural Network (ANN)}

2.2.1. Confirmation of the Structure of the Network. The ANN has received considerable attention as a powerful computational tool for forecasting in many fields since 1980. ANN models always outperform statistical models because of their ability to map the inputs onto outputs via simple computation [37]. We discuss the feed-forward neural network in this paper because of its strong learning ability and simple structure. The determination of the network structure is as follows [38].

Definition 3. Given an arbitrary continuous function $f$ : $[0,1]^{n} \rightarrow R^{m}, f$ can be accurately approximated using a three-layer forward neural network realization. The first layer of the network is the input layer, containing $n$ neurons. The middle layer is the hidden layer, containing $2 n+1$ neurons. The third layer is the output layer, which has $m$ neurons.
Definition 4. Let $\phi$ be bounded continuous monotone in function, let $K$ be the bounded compact subsets, and let $K \subset R^{n}, f(X)=f\left(x_{1}, x_{2}, \ldots, x_{n}\right)$ be a real continuous function in $f(X) \in K . \forall \varepsilon>0, \exists$ integer $N$, real constant $C_{i}, \theta_{i}(i=1,2, \ldots, N)$ and $W_{i j}(i=1,2, \ldots, N)$, such that $\widehat{f}_{i j}\left(x_{1}, x_{2}, \ldots x_{n}\right)=\sum_{i=1}^{N} C_{i} \phi\left(\sum_{j=1}^{n} W_{i j} x_{j}-\theta_{i}\right)$ satisfying $\max \left|\widehat{f}\left(x_{1}, \ldots, x_{n}\right)-f\left(x_{1}, \ldots, x_{n}\right)\right|<\varepsilon$. Note that, $\forall \varepsilon>0$, $\exists$ a three-layer network structure, and the output function of the hidden layer is $\phi(x)$, the output function of the input and output layer is linear, and the total relationship of $I / 0$ is $f\left(x_{1}, \ldots, x_{n}\right)$, such that $\max \mid \widehat{f}\left(x_{1}, \ldots, x_{n}\right)-f\left(x_{1}, \ldots\right.$, $\left.x_{n}\right) \mid<\varepsilon$.

Proof. Because $f(X)\left(X=\left(x_{1}, \ldots, x_{n}\right)\right)$ is a continuous function, $f(X) \in K, K \subset R^{n}$, assume that $f(X)$ is a bounded $C^{\infty}$ function. Based on the Paley-Wiener Theorem [39], the Fourier transform $F(W)\left(W=\left(W_{1}, \ldots, W_{n}\right)\right)$ is a real analytic function of $f(X), \forall$ a constant $C_{N}$, such that $|F(W)| \leq$ $C_{N}(1+|W|)^{-N}$. We define such a function:

$$
I_{A}\left(x_{1}, \ldots, x_{n}\right)=\int_{-A}^{A} \ldots \int_{-A}^{A} \phi\left(\sum_{i=1}^{n} x_{i} W_{i}-W_{0}\right) \frac{1}{(2 \pi)^{n} \phi(1)} F\left(W_{1}, \ldots, W_{n}\right) \exp \left(i W_{0}\right) d W_{0} \ldots d W_{n}
$$




$$
\begin{aligned}
I_{\infty, A}\left(x_{1}, \ldots, x_{n}\right) & =\int_{-A}^{A} \ldots \int_{-A}^{A}\left[\int_{-\infty}^{\infty} \phi\left(\sum_{i=1}^{n} x_{i} W_{i}-W_{0}\right) \frac{1}{(2 \pi)^{n} \phi(1)} F\left(W_{1}, \ldots, W_{n}\right) \exp \left(i W_{0}\right)\right] d W_{0} \cdots d W_{n}, \\
J_{A}\left(x_{1}, \ldots, x_{n}\right) & =\frac{1}{(2 \pi)^{n}} \int_{-\infty}^{\infty} \ldots \int_{-\infty}^{\infty} F\left(W_{1}, \ldots, W_{n}\right) \exp \left(i \sum_{i=1}^{n} x_{i} W_{i}\right) d W_{1} \cdots d W_{n}
\end{aligned}
$$

Using an estimation of $F(\cdot), \lim _{A \rightarrow \infty} J_{A}\left(x_{1}, \ldots, x_{n}\right)=$ $f\left(x_{1}, \ldots, x_{n}\right)$ can be proved: that is, $J_{A}$ is uniform convergence. Thus, $\lim _{A \rightarrow \infty} I_{\infty, A}\left(x_{1}, \ldots, x_{n}\right)=f\left(x_{1}, \ldots, x_{n}\right)$. That is, $\forall \varepsilon>0, \exists A>0$, such that $\max _{x \in R^{n}} \mid I_{\infty, A}\left(x_{1}, \ldots, x_{n}\right)-$ $f\left(x_{1}, \ldots, x_{n}\right) \mid<E / 2$. Moreover, for $A^{\prime}>0$, let $I_{A^{\prime}, A}\left(x_{1}, \ldots, x_{n}\right)=\int_{-A}^{A} \cdots \int_{-A}^{A}\left[\int_{-A^{\prime}}^{A} \phi\left(\sum_{i=1}^{n} x_{i} W_{i}-W_{0}\right) \cdot(1 /\right.$ $\left.\left.(2 \pi)^{n} \phi(1)\right) F\left(W_{1}, \ldots, W_{n}\right) \cdot \exp \left(i W_{0}\right)\right] d W_{0} \cdots d W_{n}$. For $\varepsilon>0$, $A^{\prime}>0$ can be found such that $\max _{x \in K} \mid I_{A, A}\left(x_{1}, \ldots, x_{n}\right)-$ $I_{\infty, A}\left(x_{1}, \ldots, x_{n}\right) \mid<\varepsilon / 2$. That is to say, $f(X)$ is a uniform approximation by the integral $I_{A^{\prime}, A}(X), I_{A^{\prime}, A}(X) \in K$. If $A_{i}>0(i=1,2, \ldots, m), K$ is a bounded subset, $K \subset R^{n}$, and $h_{i}\left(x_{1}, \ldots, x_{m} ; t_{1}, \ldots, t_{m}\right)$ is a uniformly continuous function of $\left[-A_{1}, A_{1}\right] \times \cdots \times\left[-A_{m}, A_{m}\right] \times K$. There are functions:

$$
\begin{aligned}
& H(t)=\int_{-A_{1}}^{A_{1}} \cdots \int_{-A_{m}}^{A_{m}} h\left(x_{1}, \ldots, x_{m} ; t_{1}, \ldots, t_{m}\right) d x_{1} \\
& \cdots d x_{m} \\
& H_{N}(t)=\frac{2 A_{1} \cdots 2 A_{m}}{N^{m}} \times \sum_{k_{1} \cdots k_{m}=0}^{N-1} h\left(-A_{1}\right. \\
& \left.+\frac{k_{1} \cdot 2 A_{1}}{N} \cdots A_{m}+\frac{k_{m} \cdot 2 A_{m}}{N} t_{1} \cdots t_{m}\right) .
\end{aligned}
$$

$\forall \varepsilon>0, \exists N_{0}, N_{0}$ is a natural number, when $N \geq N_{0}$, such that

$$
\max _{l \in K}\left|H(t)-H_{N}(t)\right|<\varepsilon
$$

Thus, $I_{A^{\prime}, A}(X)$ is a uniform approximate on $K$,

$$
\begin{aligned}
\phi\left(\sum_{i=0}^{n} x_{i} W_{i}-W_{0}\right)= & \phi\left(\sum_{i=1}^{n} \frac{W_{i} x_{i}}{\delta}-W_{0}+\alpha\right) \\
& -\phi\left(\sum_{i=1}^{n} \frac{W_{i} x_{i}}{\delta}-W_{0}-\alpha\right) .
\end{aligned}
$$

The formula above can be achieved by a three-layer neural network. Thus, $f(X)$ can be approximated by that neural network.

Definition 5. $\forall \varepsilon>0$ and $f:[0,1]^{n} \rightarrow R^{m}$, there exists a three-layer structure that can approximate $f$ in any square error precision of $\varepsilon$.

The definition above proves that, $\forall f:[0,1]^{n} \rightarrow R^{m}$, we can use a feed-forward neural network with a three-layer structure $n \times(2 n+1) \times m$ to approximate it accurately.
Thus, this part not only proves the existence of the mapping network but also demonstrates the network structure of the mapping. In summary, this paper adopts the three-layer neural network as the basis neural network.

2.2.2. BPNN. BPNN is a type of multilayer feed-forward neural network with an error back propagation learning process. The structure of BPNN is illustrated in Figure 2. Details of $\mathrm{BPNN}$ are introduced in [40].

2.2.3. WNN. WNN, a feed-forward network, is generally multilayer [41]. It is widely applied in signal processing because of its advantages of the localization property and generalization ability [42]. The structure of WNN is shown in Figure 2.

2.3. Neural Network Optimized by an Intelligence Algorithm. The intelligent optimization algorithm provides an efficient and powerful mathematical tool for optimizing the initial weights and thresholds of the ANN [43].

2.3.1. CSA. Cuckoo search is a heuristic swarm intelligence algorithm inspired by the behavior of the obligating brood parasitism of cuckoo species [44]. CSA is utilized in this paper for its stronger capability of global optimization [43].

Definition 6. To simulate the mode of cuckoo breeding, three idealized assumptions are presented, as follows: (1) each cuckoo selects nest randomly and dumps only one egg at a time, (2) the eggs with high quality will be carried over to the next generation, and (3) the available nest number $n$ is fixed, and the probability of the host bird discovering the exotic egg is $p_{\alpha}$.

Definition 7. The Lévy flight model simulates the process of the nest-seeking characteristic of cuckoo, and the update formula of the path and location is as follows:

$$
X_{i}^{\text {iter+1 }}=X_{i}^{\text {iter }}+\alpha \oplus L(\lambda)
$$

$X_{i}^{\text {iter }}$ represents the location of nest $i$ at generation iter, $\alpha$ is the step length-controlled factor, $\oplus$ is the point-to-point multiplication, and $\operatorname{Levy}(\lambda)$ obeys the Lévy distribution with a random search path of parameter $\lambda$ :

$$
L(\lambda)=0.01 \frac{u}{|v|}\left(X_{i}^{\mathrm{iter}}-X_{*}^{\mathrm{iter}}\right) .
$$




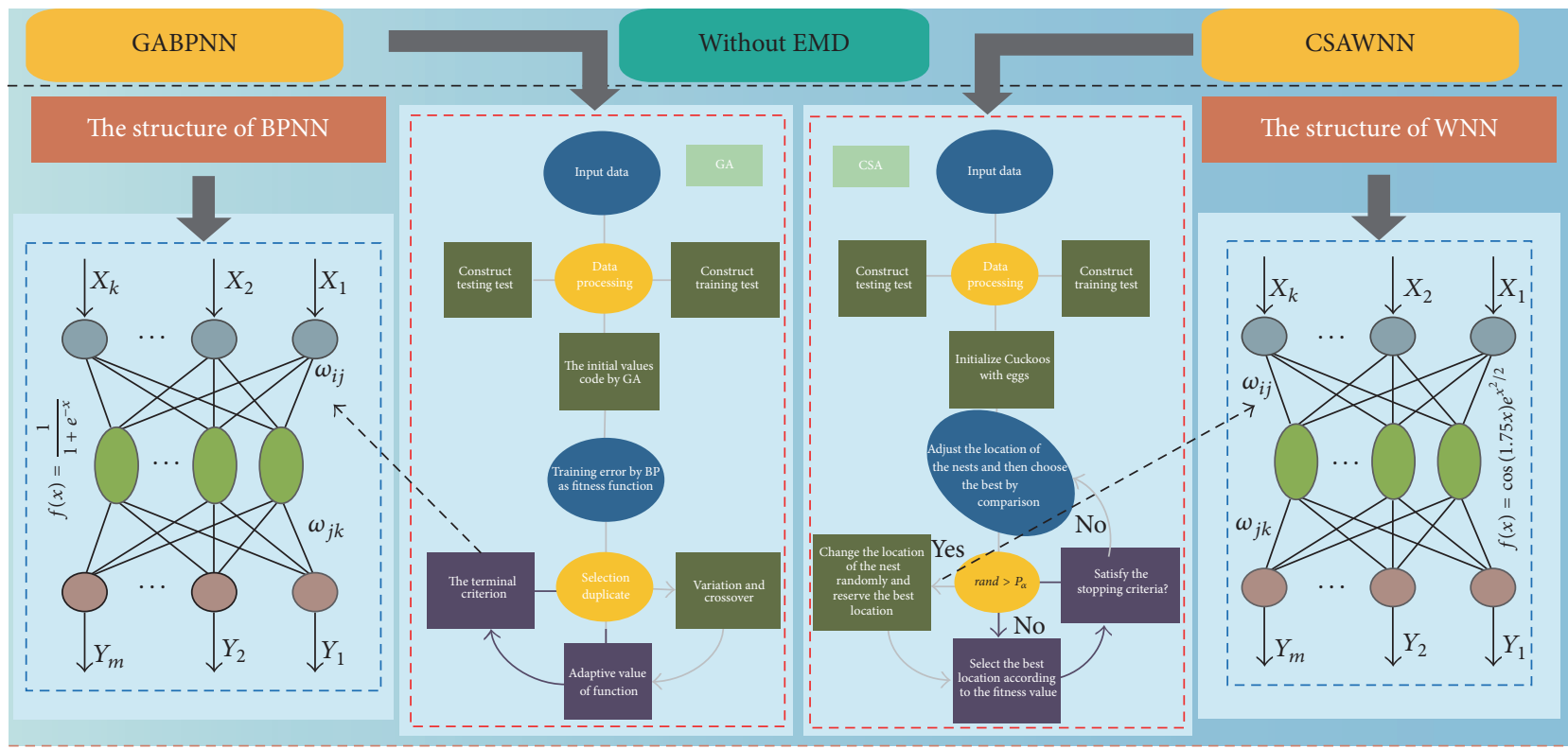

FIgURE 2: The construction of GABPNN and CSAWNN.

Here $u=t^{-\lambda}, 1<\lambda<3, u$ and $v$ obey the normal distribution, $u \sim N\left(0, \sigma_{u}^{2}\right), v \sim N(0,1), \sigma_{u}=[\Gamma(\lambda) \sin (0.5 \pi(\lambda-1)) /$ $\left.2^{(\lambda-2) / 2} \Gamma(0.5 \lambda)(\lambda-1)\right]^{1 /(\lambda-1)}, X_{*}^{\text {iter }}$ denotes the location of the best nest at generation iter, and $\Gamma$ is the standard gamma function with unbounded variance and mean of the probability distribution.

2.3.2. GA. GA is a population-based optimization algorithm that simulates natural genetic mechanisms and biological evolutionism. It possesses a capacity for powerful global optimization [45]. The principle of GA relies on a random process, which is constituted by the processes of selection, crossover, and mutation [46]. The implementation process is shown in Figure 2.

2.4. GABPNN. The primary mechanism of GABPNN is composed of three parts: GA optimization, determination of the BPNN structure, and forecasting covered by BPNN [47]. The pseudo-code for GABPNN is as shown in Algorithm 1.

2.5. EMD-CSAWNN. In this paper, the proposed model, which incorporates the empirical model decomposition technique into the WNN model based on CSA, is adopted for short-term power load forecasts. Empirical model decomposition represents a self-adaptive decomposition technique to decompose short-term power load series into several IMFs and one residual item. WNN is adopted as a forecasting engine in the proposed approach because of its powerful approximation and high computation speed. Additionally, to avoid the deficiencies of WNN such as its unstable structure, CSA is used to initialize and determine the weights and thresholds of WNN, thereby imparting an outreach capacity to WNN. Figure 3 illustrates the general structure of the hybrid power load forecasting method. The pseudo-code for the algorithm of the EMD-CSAWNN model is as shown in Algorithm 2.

\section{Experiments and Evaluations}

Applications of the proposed hybrid approach and five comparison models are shown in this subsection. All algorithms are operated on the given platform: $3.20 \mathrm{GHz}$ CPU, 8.00 GB RAM, Windows 7, and MATLAB R2012a. Meanwhile, taking into account the randomness factors and to make sure the final results are reliable and independent from the initial weights, we carry out each ANN experiment 50 times and then take the average value.

3.1. Region Description and Data Collection. Australia has plentiful coal and wind resources across its coastline and land. A power load data set from NSW, which is the state with the largest population and highest levels of industrialization and urbanization in Australia, is employed to validate each model. In this paper, power load data are collected randomly from NSW from January 12, 2009, to March 8, 2009, which includes eight weeks of data. Among them, the data from January 12, 2009, to February 1, 2009, is used as a training set to obtain the appropriate model, and data from February 2, 2009, to March 1, 2009, is used as the testing set. The power load data from January 12, 2009, to February 1, 2009, together with their statistical measures, that is, minimum, maximum, mean, and standard deviations, are shown in Figure 4(c); the standard deviations are all above 1800, which implies that the power load series fluctuates significantly with the minimum/ maximum of week one, week two, and week three, which are 6049.94/13518.06 (MWh), 6280.27/13326.47, and 6375.84/ 13096.37 , respectively. This can be intuitively observed from the amplitude and frequency of the series fluctuation, which can change from very high to low values and vice versa. 
(1) GENERATE iter $=0 \quad I^{*}$ Calculate the fitness of each individual in the population. ${ }^{*} /$

(2) DO WHILE iter $\leq$ iter $_{\max }$

(3) iter $=$ iter $+1 \quad I^{*}$ Record the best fitness values and the average fitness values. $* /$

(4) FOR EACH $1 \leq i \leq P$ DO $/{ }^{*}$ The process of the select operation. * / $p_{i}^{\text {iter }}=f_{i}^{\text {iter }}(\cdot) / \sum_{i=1}^{P} f_{i}^{\text {iter }}(\cdot)$

FOR EACH $1 \leq j \leq s$ DO $/{ }^{*}$ The process of the crossover and mutation operations. */

$$
\begin{aligned}
& w_{k j}^{\text {iter }}=w_{k j}^{\text {iter }}(1-b)+w_{l j}^{\text {iter }} b w_{l j}^{\text {iter }}=w_{l j}^{\text {iter }}(1-b)+w_{k j}^{\text {iter }} b \\
& w_{i j}^{\text {iter }}= \begin{cases}w_{i j}^{\text {iter }}+\left(w_{i j}^{\text {iter }}-w_{\max }^{\text {iter }}\right) r_{2}\left(1-\text { iter } / G_{\max }\right) & r \geq 0.5 \\
w_{i j}^{\text {iter }}+\left(w_{\text {min }}^{\text {iter }}-w_{i j}^{\text {iter }}\right) r_{2}\left(1-\text { iter } / G_{\max }\right) & r<0.5\end{cases}
\end{aligned}
$$

\section{(9) END FOR; END WHILE}

(10) $W_{\text {best }}=\left[\begin{array}{llll}\omega_{j k} & \omega_{i j} & a_{j} & b_{k}\end{array}\right] /{ }^{*}$ Initial weights and thresholds of BPNN by the obtained best individual. */

(11) $x_{k}=\left(x_{k}-x_{\min }\right) /\left(x_{\max }-x_{\min }\right), k \in[1, q] \quad /^{*}$ The process of training data normalization. * /

(12) iter $=0 /{ }^{*}$ Adjust the weights and threshold of BPNN according to the forecast error. ${ }^{*} /$

(13) DO WHILE iter $\leq$ iter $_{\max }$

(14) $\quad$ iter $=$ iter +1

(15) $\quad$ FOR EACH $1 \leq i \leq n$ DO

(16) $\quad$ FOR EACH $1 \leq j \leq n_{\text {Hidden }}$ DO

(17)

(24) END FOR; END WHILE $H_{j}=f\left(\sum_{i=1}^{n} \omega_{i j} x_{i}-a\right) \quad I^{*}$ Calculate the outputs of the hidden layer. ${ }^{*} /$ FOR EACH $1 \leq k \leq m$ DO

$$
\begin{aligned}
& O_{k}=\sum_{j=1}^{l} H_{j} \omega_{j k}-b_{k} I^{*} \text { Calculate the outputs of the output layer. */ } \\
& e_{k}=y n(k)-O_{k} I^{*} \text { Calculate the forecast error in the output layer. */ } \\
& \omega_{i j}=\omega_{i j}+\eta H_{j}\left(1-H_{j}\right) x(i) \sum_{k=1}^{m} \omega_{j k} e_{k} I^{*} \text { Update the connection weights */ } \\
& \omega_{j k}=\omega_{j k}+\eta H_{j} e_{k} \\
& a_{j}=a_{j}+\eta H_{j}\left(1-H_{j}\right) \sum_{k=1}^{m} \omega_{j k} e_{k} b_{k}=b_{k}+e_{k} I^{*} \text { Update the threshold. */ }
\end{aligned}
$$

(25) RETURN $\hat{x}_{f}=(\widehat{x}(q+1), \hat{x}(q+2), \ldots, \hat{x}(q+p))$

Algorithm 1: GABPNN.

Figure 4(b) exhibits 1008 power load data points from January 12, 2009, to February 1, 2009, divided into three groups, with 336 data points in every group. Because the power load data of NSW are collected once every half hour, each day includes 48 data points. On different days of the week, daily life and human economic production usually have different behaviors; thus, the characteristics of the load are different on different days. To minimize forecasting error as much as possible, we forecast the load of different days in the week separately. In this paper, the cycles of data division are seven days; the first three weeks of Monday data, January 12, January 19, and January 26, are employed to forecast the next Monday load on February 2, 2009. Accordingly, the data on January 13, January 20, and January 27 are employed to forecast the power load on February 3, 2009. The rest can be conducted in the same manner. The structures of the training and testing sets are illustrated in Figure 3(b).

3.2. Evaluation Metrics. Forecasting accuracy is an important criterion for evaluating a forecasting model. In this paper, the basic error calculation method is as follows:

$$
\mathrm{MAE}=\frac{1}{T} \sum_{t=1}^{T}\left|\mathrm{AE}_{t}\right|=\frac{1}{T} \sum_{t=1}^{T}\left|x_{t}-\widehat{x}_{t}\right| .
$$

Here, $T$ is the number of data points; the formula AE represents the absolute error between the observed value and the forecasting value at time $t, x$ is the observed value, and $\hat{x}_{t}$ is the forecasting value at time $t$. To avoid a positive or negative offset in forecasting error, solve the problem for which positive and negative forecasting error cannot be added, adding to the absolute value of the error, and take the average in the end. This error belongs to the comprehensive index in error analysis:

$$
\mathrm{RMSE}=\sqrt{\frac{1}{T} \sum_{t=1}^{T} \mathrm{AE}_{t}^{2}}=\sqrt{\frac{1}{T} \sum_{t=1}^{T}\left(x_{t}-\widehat{x}_{t}\right)^{2}} .
$$

RMSE is the square root of the mean square error. It also belongs to the comprehensive index in error analysis. Take the square of the absolute error AE; thus, the role of great values in the error will be strengthened, improving the sensitivity of the indicators, which is the prevailing reason in the error analysis:

$$
\mathrm{MAPE}=\frac{1}{T} \sum_{t=1}^{T}\left|\frac{x_{t}-\widehat{x}_{t}}{x_{t}}\right| \times 100 \%,
$$

where the symbolic meaning is as above. The indicator is the average of the absolute error. The index is one of the comprehensive indexes in error analysis that usually occupies a very important position in the analysis and forecasting performance of the model. 


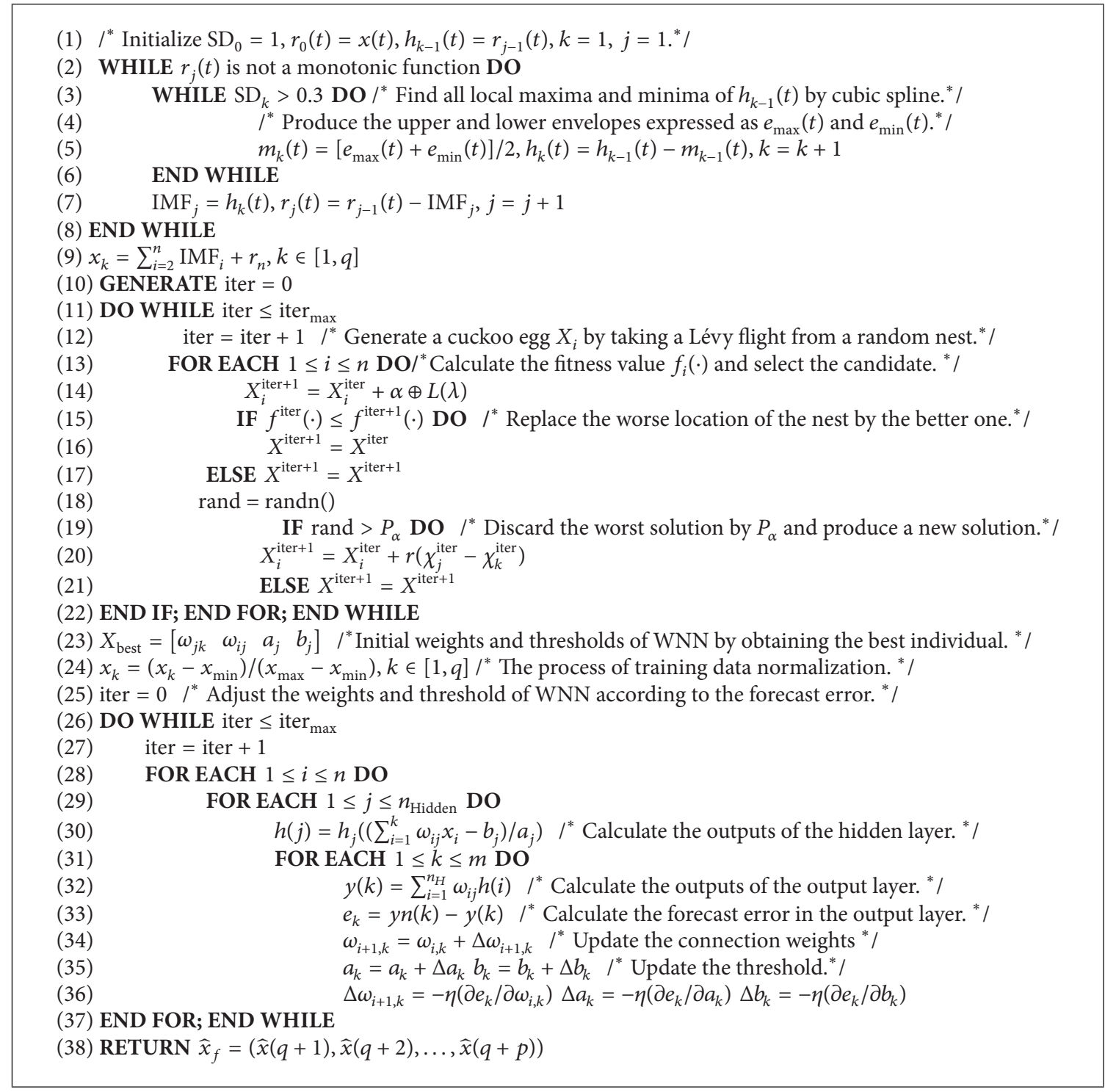

Algorithm 2: EMD-CSAWNN.

To determine the degree of correlation between different forecasting model results with observed values, GRA [48] is employed in this paper.

Definition 8 (determining the grey relational coefficient). $\left\{x_{0}(i)\right\}_{i=1}^{n}$ is the reference sequence, $\left\{x_{j}(i)\right\}_{i=1}^{n}$ is the comparative sequence, $j=1,2, \ldots, m$, and the relational coefficient of $x_{0}$ and $x_{j}$ in point $k$ is represented as

$$
\begin{aligned}
& \xi_{j}(k) \\
& =\frac{\min _{j} \min _{k}\left|x_{0}(k)-x_{j}(k)\right|+\rho \max _{j} \max _{k}\left|x_{0}(k)-x_{j}(k)\right|}{\left|x_{0}(k)-x_{j}(k)\right|+\rho \max _{j} \max _{k}\left|x_{0}(k)+x_{j}(k)\right|} .
\end{aligned}
$$

Here, $\rho$ is the distinguishing coefficient, and $\rho \in[0,1]$, usually $\rho=0.5$.
Definition 9 (grey relational degree). By focusing the degree of $\xi_{j}(k)$ at utter points, the algorithm on the grey relational degree is

$$
r_{i}=\frac{1}{n} \sum_{k=1}^{n} \xi_{i}(k) .
$$

Considering the generation capacity of the proposed hybrid model, four statistical indices are employed as evaluation metrics to measure the forecasting accuracy, MAE, RMSE, MAPE, and GRA. MAPE, MAE, and RMSE measure the mean performance, and GRA illustrates how well the forecasted data points fit the trend.

\section{Results and Analysis}

The experiments were divided into three parts, Experiment 1, Experiment 2, Experiment 3, Experiment 4, and 

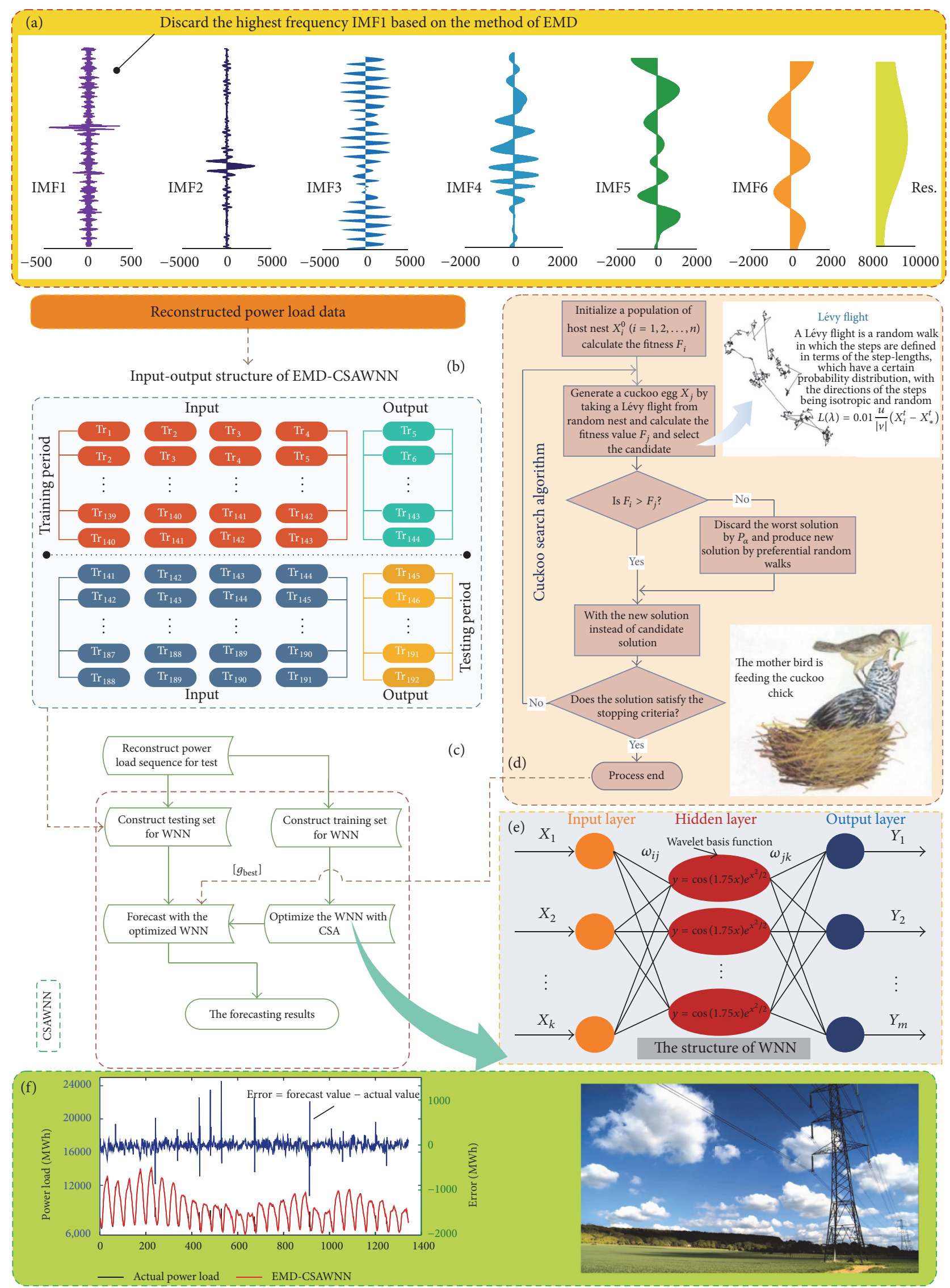

FIGURE 3: The construction of EMD-CSAWNN. (a) IMFs and the residual decomposed by empirical model decomposition. (b) The structure of the training and testing sets of the neural network. (c) The procedure of WNN combined with CSA. (d) The procedure of CSA. (e) The structure of WNN. (f) The forecasting sequence and error by using the EMD-CSAWNN model. 


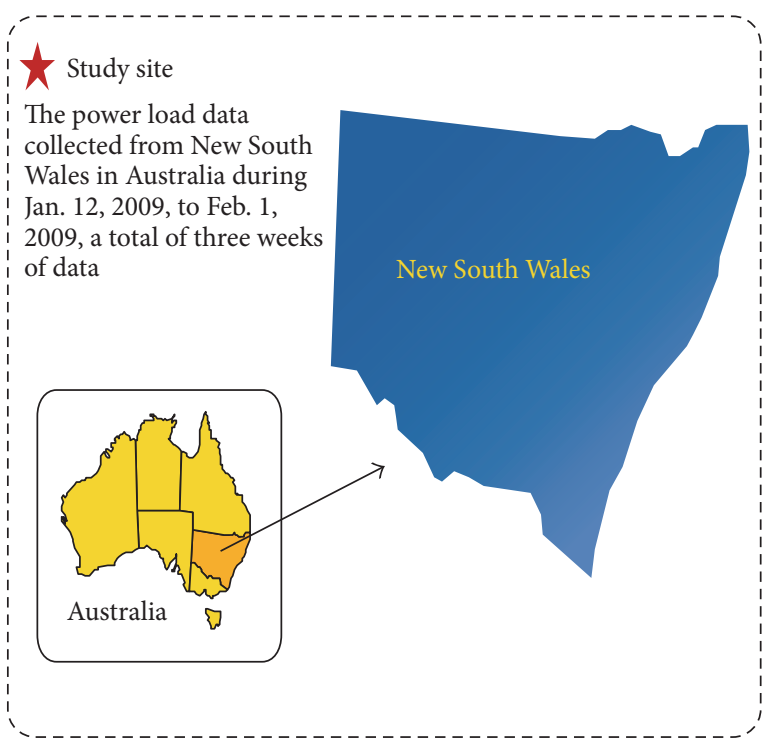

(a)

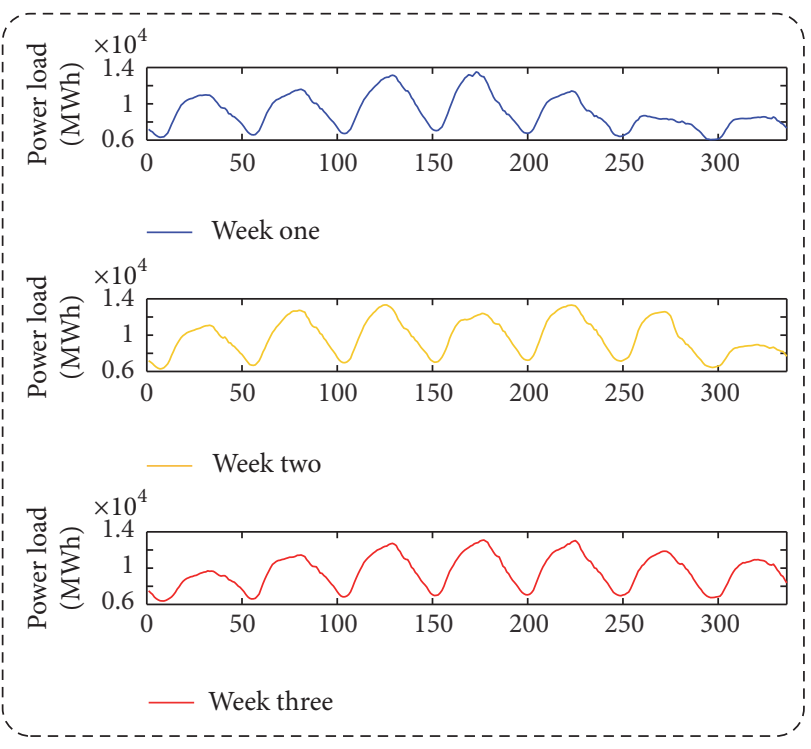

(b)

\begin{tabular}{|c|c|c|c|c|}
\hline \multirow{2}{*}{ Time } & \multicolumn{4}{|c|}{ Power load of New South Wales from Jan. 12 to Feb. 1, 2009} \\
\hline & Mean (MWh) & Standard deviation & $\operatorname{Min}(\mathrm{MWh})$ & $\operatorname{Max}(\mathrm{MWh})$ \\
\hline Week one & 9115.66 & 1895.18 & 6049.94 & 13518.06 \\
\hline Week two & 9753.53 & 2017.32 & 6280.27 & 13326.47 \\
\hline Week three & 9753.53 & 1854.42 & 6375.84 & 13096.37 \\
\hline
\end{tabular}

(c)

Figure 4: Description of observations in New South Wales of China. (a) Location of the study site. (b) The original power load series from January 12 to February 1, 2009. (c) The statistical measures for the power load.

Experiment 5. Experiment 1, the primary experiment, aims to compare the performance of different models in one day (February 2, 2009) and, meanwhile, to compare the ARIMA and ANN models. Experiment 2 aims to compare the performance of different models between weekdays and weekends. Experiment 3 aims to compare the performance of different models in 28 days (from February 2, 2009, to March 1, 2009). In order to further validate the hybrid model we proposed Experiments 4 and 5 which are studied in this paper. In addition, ENN (Elman neural network) and RBFNN (radial basis function neural network) are utilized in further comparison. Experiment 4 aims at globally testing the proposed hybrid model by using real power load data on Thursday, April 24, 2008, and Tuesday, April 29, 2008, which is selected from New South Wales of Australia randomly. Experiment 5 aims to prove the general applicability of hybrid model; thus, the power load data on Saturday, June 28, 2008, and Monday, June 30, 2008, from Victoria of Australia are chosen for forecasting.

Experiment 1. According to determination of the network structure, $n \times(2 n+1) \times m$ as the structure of the three-layer neural network will achieve better forecasting results. The network structure of the ANN model is 4-9-1 because of its four nodes in the input layer and one node in the output layer.
The iteration time is set to 100 , the learning rate is set to 0.01 , and the training requirement accuracy is set to 0.0004 . To forecast the power load on Monday, February 2, the historical values from the Mondays of the first three weeks, January 12, January 19, and January 26, are chosen. Test results (MAE, RMSE, MAPE, and GRA) are presented in Tables 1 and 2, Figures 5, 6, and 7. Each individual model exhibits its best performance at a special time. For example, Figure 6 shows that BPNN provides the lowest MAPE value at 3:00 among all of the individual models, and CSAWNN yields the highest accuracy forecasting value from 1:00 to 3:00 among all of the individual models, whereas the maximum error is with the BPNN forecasting model on February 2, with a MAPE value of $10.12 \%$. This result is due to the unstable initialization of the ANN. The result of the original data of empirical model decomposition is shown in Figure 3(a). The noise in the data is eliminated by using the empirical model decomposition technique; in this paper, the IMF1 is a high-frequency sequence with small values, which can be regarded as interference factors. As a result, the rest of the IMFs and the remainder term can be constructed as the training input of the CSAWNN model. Table 1 shows the experimental results for Monday of six types of forecasting models. The average values of MAPE for six models on February 2, 1.6\% are 1.54\%, 1.97\%, $1.37 \%, 1.02 \%$, and $1.94 \%$, respectively; as shown in Table 2, 


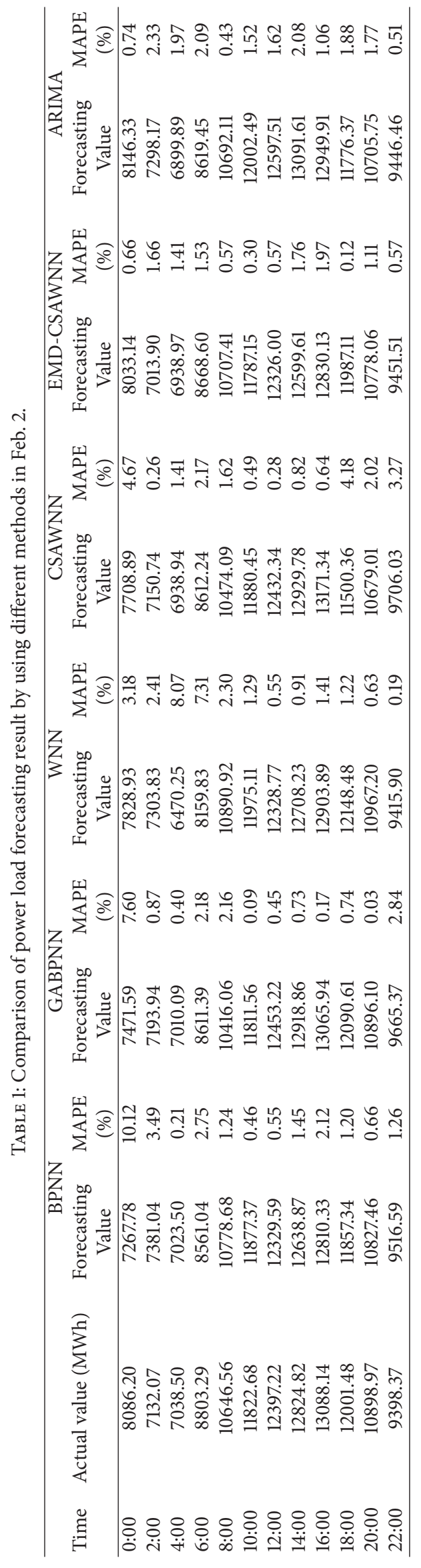




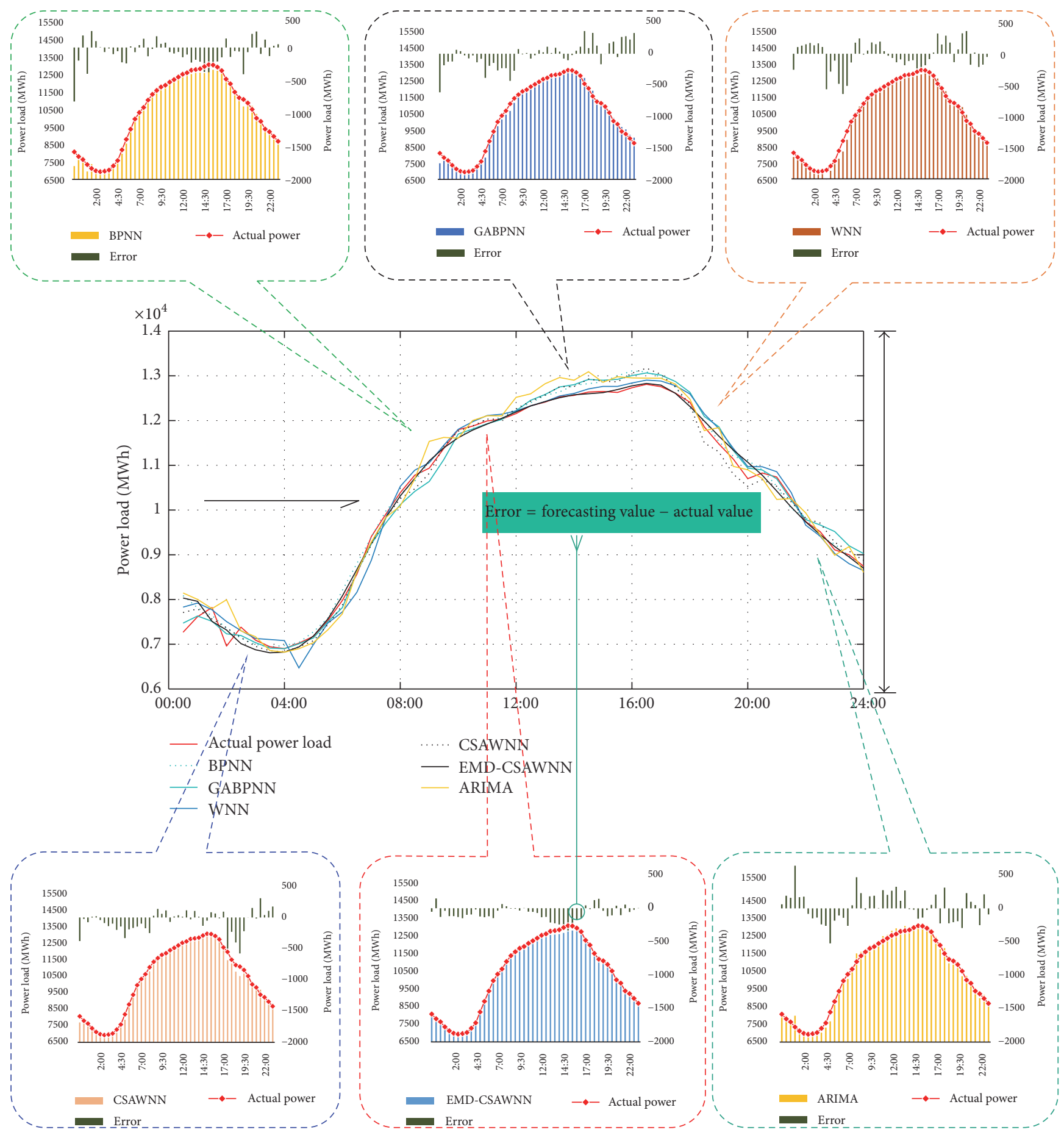

FIGURE 5: The forecasting results and actual values for February 2.

the MAPE afforded by the hybrid model decreased by $48.22 \%$ compared with the maximum average value of MAPE. In addition, it is shown that the value of MAPE offered by the hybrid model is more stable than that of the other proposed models, and the maximum value of MAPE is $2.54 \%$. By comparing the hybrid model with the other models, it is shown that the hybrid model can provide high and stable forecasting accuracy.
Experiment 2. Figure 8 and Table 3 describe the comparison of six models with values on weekdays and weekends on different evaluation metrics. On weekdays, the best performance model is the hybrid model and the value of MAPE is $0.82 \%$; on the contrary, the worst is the ARIMA model, whose value of MAPE is $1.48 \%$. The MAPE offered by the hybrid model is $44.59 \%$ lower than that offered by the ARIMA model. On weekends, the value of MAPE offered by the hybrid model 
TABLE 2: Comparison of power load forecasting result by using different methods in Feb. 2.

\begin{tabular}{|c|c|c|c|}
\hline \multirow{2}{*}{ Model } & \multicolumn{3}{|c|}{ MAPE (\%) } \\
\hline & Maximum & Minimum & Average \\
\hline BPNN & 10.12 & 0.05 & 1.6 \\
\hline GABPNN & 7.6 & 0.03 & 1.54 \\
\hline WNN & 8.07 & 0.12 & 1.97 \\
\hline CSAWNN & 5.19 & 0.01 & 1.37 \\
\hline EMD-CSAWNN & 2.54 & 0.03 & 1.02 \\
\hline ARIMA & 8.67 & 0.03 & 1.94 \\
\hline
\end{tabular}

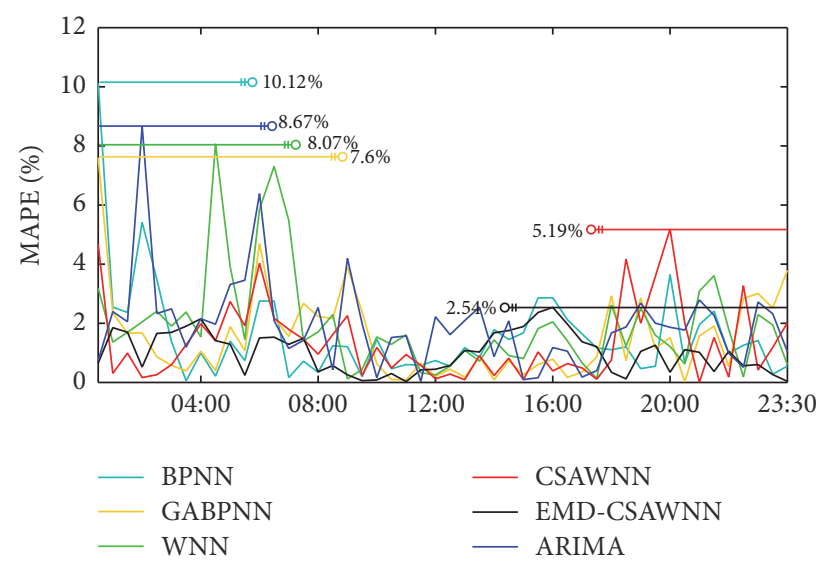

FIgURE 6: The BPNN, GABPNN, WNN, CSAWNN, EMDCSAWNN, and ARIMA forecasting MAPE (\%) for February 2.

is $0.68 \%$, which outperforms all other models and is $49.25 \%$ lower than that of the worst model, ARIMA. Figure 8 indicates that, except for the criterion RMSE, the weekdays outperform the weekends when utilizing WNN and the criterion GRA, the weekdays outperform the weekends when utilizing the BPNN, GABPNN, WNN, and CSAWNN models, and the other performances on weekends are all better than those on weekdays when utilizing the six models. The values of MAPE offered by the six models on weekends are $1.23 \%, 1.05 \%$, $1.27 \%, 1.13 \%, 0.68 \%$, and $1.34 \%$, which are $5.38 \%, 10.25 \%$, $0.78 \%, 8.13 \%, 17.07 \%$, and $9.46 \%$ lower than the corresponding weekday values, respectively. This illustrates that the forecasting results on weekends outperform those of weekdays.

Experiment 3. Figures 9 and 10 illustrate the forecasting results of Experiment 3. In comparison with the BPNN, WNN, GABPNN, CSWNN, and ARIMA models, the forecasting results offered by the hybrid model are more accurate. The forecasting results are shown in Figure 10, and detailed prediction results are shown in Table 4 . The maximum values of MAPE offered by the six models are $1.28 \%, 1.14 \%, 1.27 \%$, $1.2 \%, 0.78 \%$, and $1.44 \%$, respectively. The maximum value of MAPE offered by the ARIMA model is $4.4 \%$ over 28 days. Meanwhile, on all days of the test, the average values of MAE, MAPE, and RMSE offered by the hybrid model are all smaller than those of the other models. The average value of MAPE offered by the hybrid model over four weeks is $0.78 \%$, and the highest decrease is $39.06 \%$ compared with the other ANN models. This indicates that the hybrid model is an effective power load forecasting approach. The GRA result is shown in Table 5. In addition, on March 1, the GRA of GABPNN is higher than that of the hybrid model; on the remaining 27 days, the values of GRA offered by the hybrid model are higher than those of the other five models. According to the average value of GRA over the 28 days, the forecasting effects of all six forecasting models are increasing in the following order: ARIMA, BPNN, which is the WNN, CSAWNN, GABPNN, and EMD-CSAWNN, which concludes that the effect of the hybrid forecasting model is the best model among the six forecasting models.

The higher the power load forecasting accuracy, the lower the economic cost, which has actual economic significance [49]. As is illustrated in this case, the ANN optimized by the intelligence algorithm after denoising provides a better power load forecasting effect.

Experiment 4. The power load on Thursday, April 24, 2008, and Tuesday, April 29, 2008, from New South Wales of Australia is used to globally testing the proposed hybrid model. The results are shown as in Tables 6 and 7. To forecast the power load on Thursday, April 24, 2008, the historical values from the Thursdays of the first three weeks, April 3, April 10, and April 17, are chosen, respectively. To forecast the power load on Tuesday, April 29, 2008, the historical values from the Thursdays of the first three weeks, April 8, April 15, and April 22 , are chosen, respectively. Test results (AE, MAE, RMSE, and MAPE) are presented in Tables 6 and 7 and part (a) of Figure 11. Table 6 indicates that EMD-CSAWNN has the highest accuracy forecasting results on Thursday, April 24, 2008; the maximum, minimum, and average MAPE values are $1.2355 \%$ at $2: 00,1.1836$ at $14: 00$, and $1.2025 \%$, respectively. The second-highest to sixth-highest accurate models are GABPNN, BPNN, RBFNN, CSAWNN, WNN, ENN, and ARIMA with average MAPE values of $1.6436 \%, 1.7795 \%$, $1.8314 \%, 2.3373 \%, 2.4141 \%, 3.1381 \%$, and $5.5872 \%$, respectively. Table 7 indicates that EMD-CSAWNN still yields the highest accuracy forecasting value from among all of other models mentioned in this paper when forecasting power load on Tuesday, April 29, 2008; the maximum, minimum, and average MAPE values are $1.9844 \%$ at $14: 00,1.6033 \%$ at $12: 00$, and $1.7811 \%$, respectively. According to the average MAPE value, CSANN is the second most accurate model, GABPNN is the third most accurate model, RBFNN is the fourth most accurate model, WNN is the fifth most accurate model, BPNN is the sixth most accurate model, ENN is the fifth most accurate model, and ARIMA is the sixth most accurate model with average MAPE values of $2.5827 \%, 2.8472 \%, 2.8473 \%$, $3.1717 \%, 3.2725 \%, 3.5151 \%$, and $3.7549 \%$, respectively. As shown in Table 6, the average MAPE afforded by the hybrid model decreased by $78.48 \%$ compared with the maximum average MAPE value. In Table 7, the average MAPE afforded by the hybrid model decreased by $52.57 \%$ compared with the maximum average MAPE value. In addition, it is shown that the value of MAPE offered by the hybrid model is more stable than that of the other proposed models. By comparing 


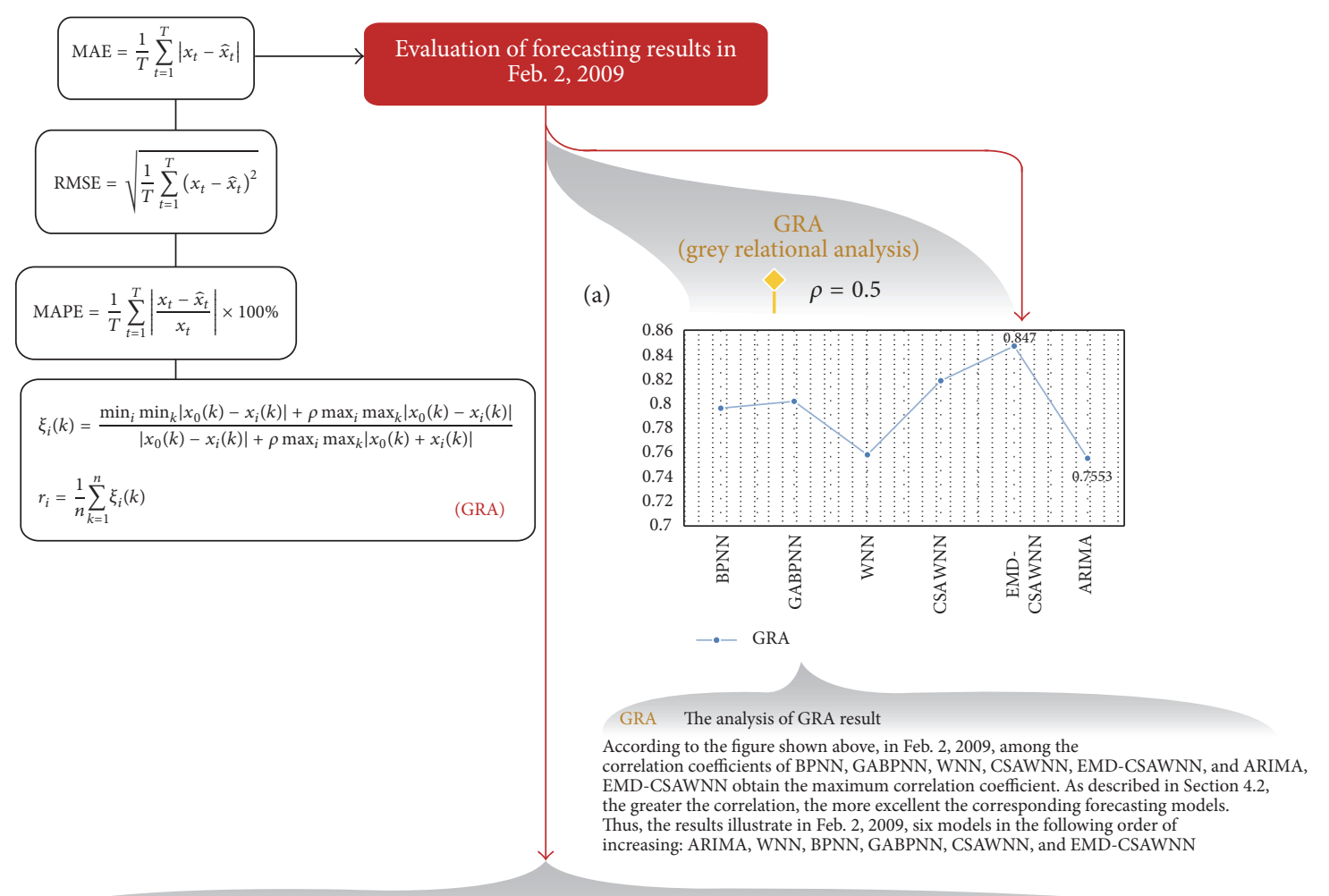

The forecasting versus actual levels

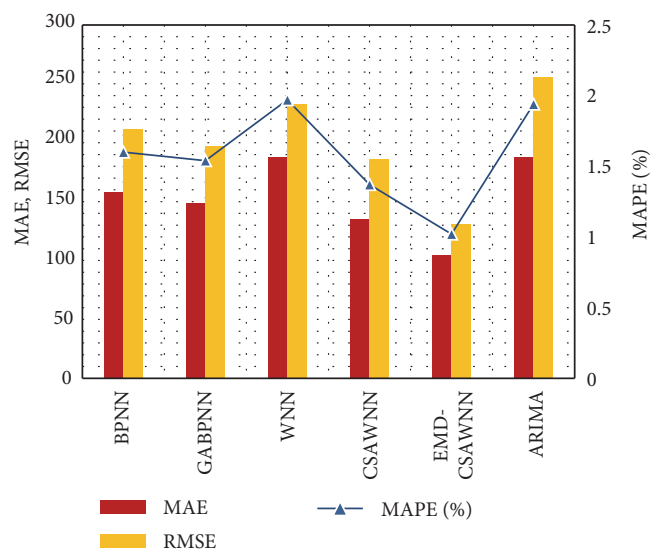

(b)

\begin{tabular}{lc}
\hline Model & MAE \\
\hline BPNN & 158.423 \\
GABPNN & 18.608 \\
WNN & 187.421 \\
CSAWNN & 134.919 \\
EMD-CSAWNN & $\mathbf{1 0 4 . 7 1 6}$ \\
\hline
\end{tabular}
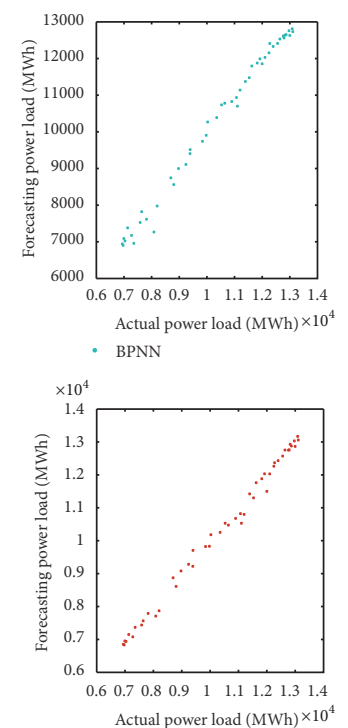

(c)
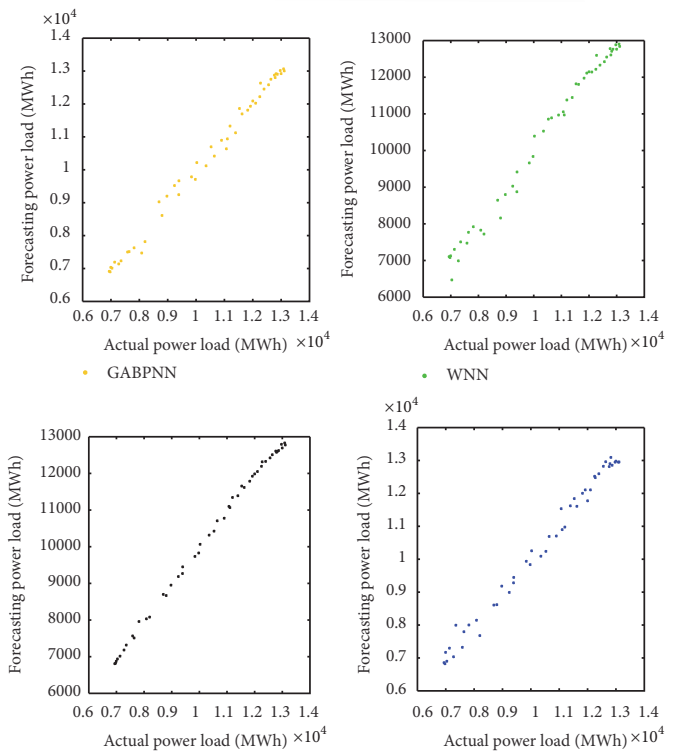

- EMD-CSAWNN

- ARIMA

\begin{tabular}{ccc}
\multicolumn{2}{c}{ Evaluation metrics in Feb. 2, 2009 } & GRA \\
RMSE & MAPE (\%) & 0.7962 \\
211.5302 & 1.6 & 0.8018 \\
196.9762 & 1.54 & 0.7582 \\
233.0043 & 1.97 & 0.8186 \\
186.0761 & 1.37 & $\mathbf{0 . 8 4 7}$ \\
$\mathbf{1 3 0 . 6 4 3 9}$ & $\mathbf{1 . 0 2}$ & 0.7553 \\
2559717 & 1.94 &
\end{tabular}

FIGURE 7: The comprehensive evaluation of forecasting models for February 2. (a) The comparison of GRA by using six different models. (b) The comparison of MAE, RMSE, and MAPE by using six different models. (c) The scatterplot of forecasting versus actual levels by using six different models. (d) The evaluation results of six different models. The value in bold is the best value of each evaluation index. 
TABLE 3: Comparison of power load forecasting evaluation by using different methods between weekdays and weekends.

\begin{tabular}{|c|c|c|c|c|c|}
\hline \multirow{2}{*}{ Model } & \multirow{2}{*}{ Date } & \multicolumn{4}{|c|}{ Evaluation criterion } \\
\hline & & MAPE (\%) & RMSE & MAE & GRA \\
\hline \multirow{2}{*}{ BPNN } & Weekdays & 1.30 & 164.97 & 118.03 & 0.8654 \\
\hline & Weekends & 1.23 & 148.35 & 101.40 & 0.8600 \\
\hline \multirow{2}{*}{ GABPNN } & Weekdays & 1.17 & 154.94 & 106.05 & 0.8781 \\
\hline & Weekends & 1.05 & 137.21 & 88.33 & 0.8779 \\
\hline \multirow{2}{*}{ WNN } & Weekdays & 1.28 & 162.09 & 114.33 & 0.8688 \\
\hline & Weekends & 1.27 & 162.83 & 108.65 & 0.8581 \\
\hline \multirow{2}{*}{ CSAWNN } & Weekdays & 1.23 & 157.05 & 109.05 & 0.8737 \\
\hline & Weekends & 1.13 & 138.86 & 94.96 & 0.8689 \\
\hline \multirow{2}{*}{ EMD-CSAWNN } & Weekdays & 0.82 & 101.56 & 72.77 & 0.9087 \\
\hline & Weekends & 0.68 & 87.00 & 55.19 & 0.9148 \\
\hline \multirow{2}{*}{ ARIMA } & Weekdays & 1.48 & 184.23 & 128.98 & 0.8539 \\
\hline & Weekends & 1.34 & 169.06 & 109.09 & 0.8548 \\
\hline
\end{tabular}

GABPNN, BPNN, CSAWNN, WNN, ARIMA, ENN, and RBFNN, it is shown that the hybrid EMD-CSAWNN model is better than that of single models.

Experiment 5. The power load on Saturday, June 28, 2008, and Monday, June 30, 2008, from Victoria of Australia is used to further prove that the proposed hybrid model can improve the performance of power load forecasting in different cases. The historical values from the Saturday of the first three weeks, June 7, June 14, and June 21, are chosen in order to forecast the power load on Saturday, June 28, 2008. Moreover, the historical values from the Monday of the first three weeks, June 9, June 16, and June 23, are chosen in order to forecast the power load on Monday, June 30, 2008. The experimental results are presented in Tables 8 and 9 and part (b) of Figure 11. For Saturday (June 28, 2008) data, the average MAPE values for the BPNN, GABPNN, WNN, CSAWNN, ARIMA, ENN, RBFNN, and EMD-CSAWNN models are 3.3224\%, $2.8656 \%, 3.2301 \%, 2.5248 \%, 3.7716 \%, 3.3890 \%, 3.1754 \%$, and $1.7699 \%$, respectively. The maximum MAPE values for the six models are $3.5467 \%$ at $16: 00,3.3061 \%$ at $18: 00,3.5751 \%$ at $4: 00,2.9527 \%$ at $16: 00,4.2174 \%$ at $0: 00,3.7591 \%$ at $20: 00$, $3.3889 \%$ at $10: 00$, and $2.0503 \%$ at $14: 00$, respectively, and the minimum MAPE values are $2.9644 \%$ at $20: 00,2.2710 \%$ at $12: 00,2.7591 \%$ at $20: 00,2.1818 \%$ at $20: 00,3.4718 \%$ at $4: 00$, $3.1379 \%$ at $18: 00,2.8405 \%$ at $22: 00$, and $1.5798 \%$ at $10: 00$, respectively. The differences between the maximum and minimum MAPE values for the models are $0.5823 \%, 1.0351 \%$, $0.816 \%, 0.1936 \%, 0.7456 \%, 0.6212 \%, 0.5484 \%$, and $0.4705 \%$. For Monday (June 30, 2008) data, the average MAPE values for the BPNN, GABPNN, WNN, CSAWNN, ARIMA, ENN, RBFNN, and EMD-CSAWNN model are 3.2747\%, 2.8415\%, $3.0806 \%, 2.5422 \%, 3.7656 \%, 3.4113 \%, 3.1982 \%$, and $1.7312 \%$, respectively. The maximum MAPE values for the six models are $3.9915 \%$ at $22: 00,3.4117 \%$ at $2: 00,3.6561 \%$ at $6: 00,2.8144 \%$ at $0: 00,4.1783 \%$ at $22: 00,3.6738 \%$ at $8: 00,3.5262 \%$ at $0: 00$, and $2.0361 \%$ at $12: 00$, respectively, and the minimum MAPE values are $3.0113 \%$ at $20: 00,2.3376 \%$ at $22: 00,2.4491 \%$ at $16: 00$, $2.1398 \%$ at $20: 00,3.2795 \%$ at $0: 00,3.2235 \%$ at $4: 00,2.9466 \%$ at $22: 00$, and $1.4521 \%$ at $10: 00$, respectively. The differences between the maximum and minimum MAPE values for the models are $0.9802 \%, 1.0741 \%, 1.207 \%, 0.6746 \%, 0.8988 \%$, $0.4503 \%, 0.5796 \%$, and $0.584 \%$. Therefore, the proposed hybrid EMD-CSAWNN model is not only the most accurate but also the most stable of the investigated forecasting models.

\section{Discussion}

In this section, we discuss two important evaluation metrics, convergence speed and degree of certainty [50], offered by the GABPNN and CSAWNN models to determine a more practical forecasting model by considering reality factors such as forecasting stability and calculation time. The results illustrate that the CSAWNN model is more practical than the GABPNN model in forecasting power load. In addition, we propose forecasting availability to analyze and evaluate the quality of power load forecasting.

5.1. Convergence Speed. The computational complexity of evolutionary algorithms and swarm intelligence still remains a challenging issue; here, we use convergence speed as one of the evaluation metrics to examine the forecasting performance of GABPNN and CSAWNN. We obtain the computation time of the best fitness by analyzing the convergence speed of GA and CSA for use in comparative evaluation of optimization algorithm performance. However, the exploration and development are always two competing goals, and the conflict would exist between the convergence speed and forecasting accuracy. We define performance less than $10^{-5}$ as the convergence criteria.

We take the data from January 12, January 19, and January 26 as an example to illustrate the convergence speed of GA and CSA; Figure 12 shows the results of the comparison of evolutions among GA and CSA with different population sizes. We observed that the fitness values monotonically decrease as the iterations increase. In addition, when the iterations are less than 100, the larger the population size, the faster the convergence speed. We also observed that CSA has better convergence speed than GA. At iteration 20, the 


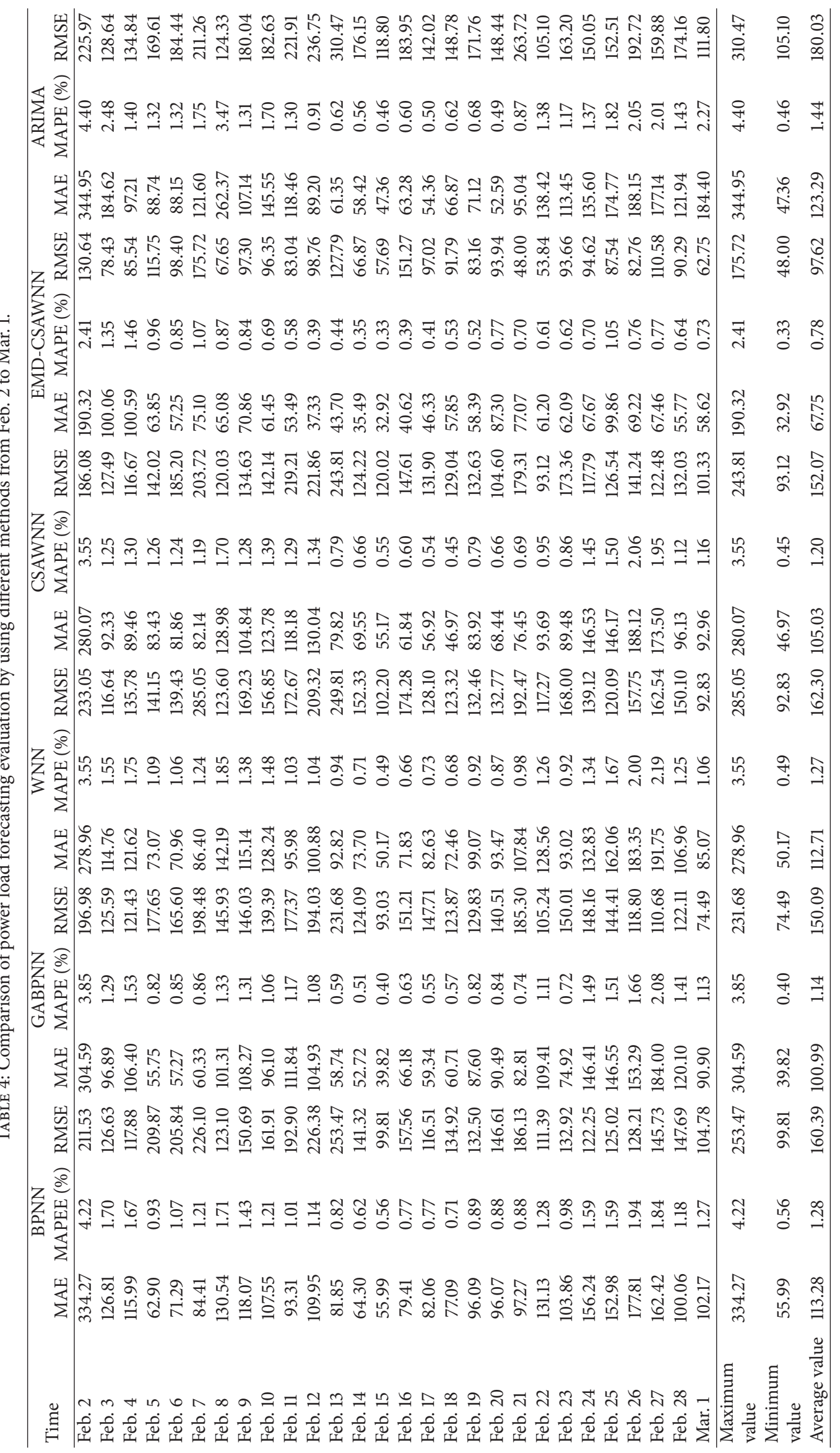


TABLE 5: Comparison of power load forecasting GRA by using different methods from Feb. 2 to Mar. 1.

\begin{tabular}{|c|c|c|c|c|c|c|}
\hline \multirow{2}{*}{ Time } & BPNN & GABPNN & WNN & CSAWN & EMD-CSAWNN & ARIMA \\
\hline & GRA & GRA & GRA & GRA & GRA & GRA \\
\hline Feb. 2 & 0.7962 & 0.8018 & 0.7582 & 0.8186 & 0.8470 & 0.7553 \\
\hline Feb. 3 & 0.7587 & 0.7532 & 0.7756 & 0.7626 & 0.8121 & 0.7468 \\
\hline Feb. 4 & 0.7132 & 0.7077 & 0.7216 & 0.7429 & 0.7611 & 0.7076 \\
\hline Feb. 5 & 0.7225 & 0.7381 & 0.7672 & 0.7717 & 0.8032 & 0.7305 \\
\hline Feb. 6 & 0.7660 & 0.7991 & 0.8194 & 0.7800 & 0.8598 & 0.8027 \\
\hline Feb. 7 & 0.8555 & 0.8680 & 0.7952 & 0.8431 & 0.8839 & 0.8381 \\
\hline Feb. 8 & 0.7507 & 0.7184 & 0.7562 & 0.7504 & 0.8191 & 0.7541 \\
\hline Feb. 9 & 0.7622 & 0.7682 & 0.7495 & 0.7840 & 0.8352 & 0.7554 \\
\hline Feb. 10 & 0.7644 & 0.8003 & 0.7750 & 0.7993 & 0.8376 & 0.7573 \\
\hline Feb. 11 & 0.8297 & 0.8608 & 0.8479 & 0.8035 & 0.9109 & 0.8032 \\
\hline Feb. 12 & 0.7949 & 0.8509 & 0.8439 & 0.8074 & 0.9018 & 0.8169 \\
\hline Feb. 13 & 0.8262 & 0.8606 & 0.8396 & 0.8493 & 0.9050 & 0.8156 \\
\hline Feb. 14 & 0.7628 & 0.8064 & 0.7800 & 0.8067 & 0.8856 & 0.8058 \\
\hline Feb. 15 & 0.7392 & 0.7565 & 0.7716 & 0.7674 & 0.8262 & 0.7317 \\
\hline Feb. 16 & 0.8147 & 0.8298 & 0.8093 & 0.8291 & 0.8520 & 0.7859 \\
\hline Feb. 17 & 0.7927 & 0.7751 & 0.7656 & 0.7755 & 0.7964 & 0.7577 \\
\hline Feb. 18 & 0.7908 & 0.8114 & 0.7938 & 0.8043 & 0.8400 & 0.7791 \\
\hline Feb. 19 & 0.8254 & 0.8199 & 0.8227 & 0.8234 & 0.8756 & 0.7884 \\
\hline Feb. 20 & 0.7249 & 0.7719 & 0.7641 & 0.7901 & 0.7907 & 0.7340 \\
\hline Feb. 21 & 0.8555 & 0.8756 & 0.8473 & 0.8676 & 0.9334 & 0.8174 \\
\hline Feb. 22 & 0.7267 & 0.7600 & 0.7412 & 0.7624 & 0.8356 & 0.7547 \\
\hline Feb. 23 & 0.8258 & 0.8255 & 0.7912 & 0.7951 & 0.8670 & 0.7872 \\
\hline Feb. 24 & 0.7511 & 0.7630 & 0.7404 & 0.7642 & 0.8316 & 0.7363 \\
\hline Feb. 25 & 0.7932 & 0.7916 & 0.8259 & 0.8200 & 0.8331 & 0.7758 \\
\hline Feb. 26 & 0.7628 & 0.7983 & 0.7316 & 0.7402 & 0.8058 & 0.7063 \\
\hline Feb. 27 & 0.7440 & 0.7870 & 0.7203 & 0.7615 & 0.8257 & 0.7306 \\
\hline Feb. 28 & 0.8012 & 0.8331 & 0.7970 & 0.8011 & 0.8666 & 0.7607 \\
\hline Mar. 1 & 0.7681 & 0.8433 & 0.7974 & 0.7667 & 0.8214 & 0.7775 \\
\hline Maximum value & 0.8555 & 0.8756 & 0.8479 & 0.8676 & 0.9334 & 0.8381 \\
\hline Minimum value & 0.7132 & 0.7077 & 0.7203 & 0.7402 & 0.7611 & 0.7063 \\
\hline Average value & 0.8673 & 0.8811 & 0.8692 & 0.8756 & 0.9127 & 0.8576 \\
\hline
\end{tabular}

convergence of CSAWNN obtained the best speed in a population of 50, and the convergence of GABPNN at the iteration between 60 and 80 , at the iteration 60 , obtained the best speed.

5.2. Degree of Certainty. The forecasting results of optimization algorithm-NN are also usually different for each experiment because of the probability mechanism of the optimization algorithm. However, in the actual forecasting field, the future values are not known; thus, we cannot obtain which experiment will obtain the best result. Hence, we use these evaluation metrics to determine the randomness.

We defined the degree of certainty as

$$
\mathrm{DC}(\sigma)=\frac{\sqrt{\sum_{i=1}^{m}\left(\sigma_{i}-\bar{\sigma}\right)^{2}}}{m},
$$

where $m$ is the number of experiments, $\sigma_{i}$ is the value of $i$ th forecasting experiment on $\sigma$, and $\bar{\sigma}$ is the average value of all $n$ experiments. It is clear that a smaller DC can bring a higher degree of certainty.

The scatterplot in Figure 13 indicates the MAPE and GRA distributions of different results of 100 experiments for February 2 by, respectively, using GABPNN and CSAWNN. Although the minimum value of MAPE of GABPNN is smaller than that of CSAWNN and $\mathrm{DC}(\sigma)$ of CSAWNN, both are smaller than those of GABPNN. DC (MAPE) and DC (GRA) of CSAWNN are 0.0014 and 0.00016 , and the DC (MAPE) and DC (GRA) of GABPNN are 0.0016 and 0.00019 . Thus, CSAWNN is a better forecasting method than GABPNN in the actual forecasting field.

5.3. Forecasting Availability. Forecasting availability can be measured not only by the square sum of forecasting error but also by the mean and mean squared deviation of the forecasting accuracy. In certain practical circumstances, the skewness and kurtosis of the distribution of forecasting accuracy need 


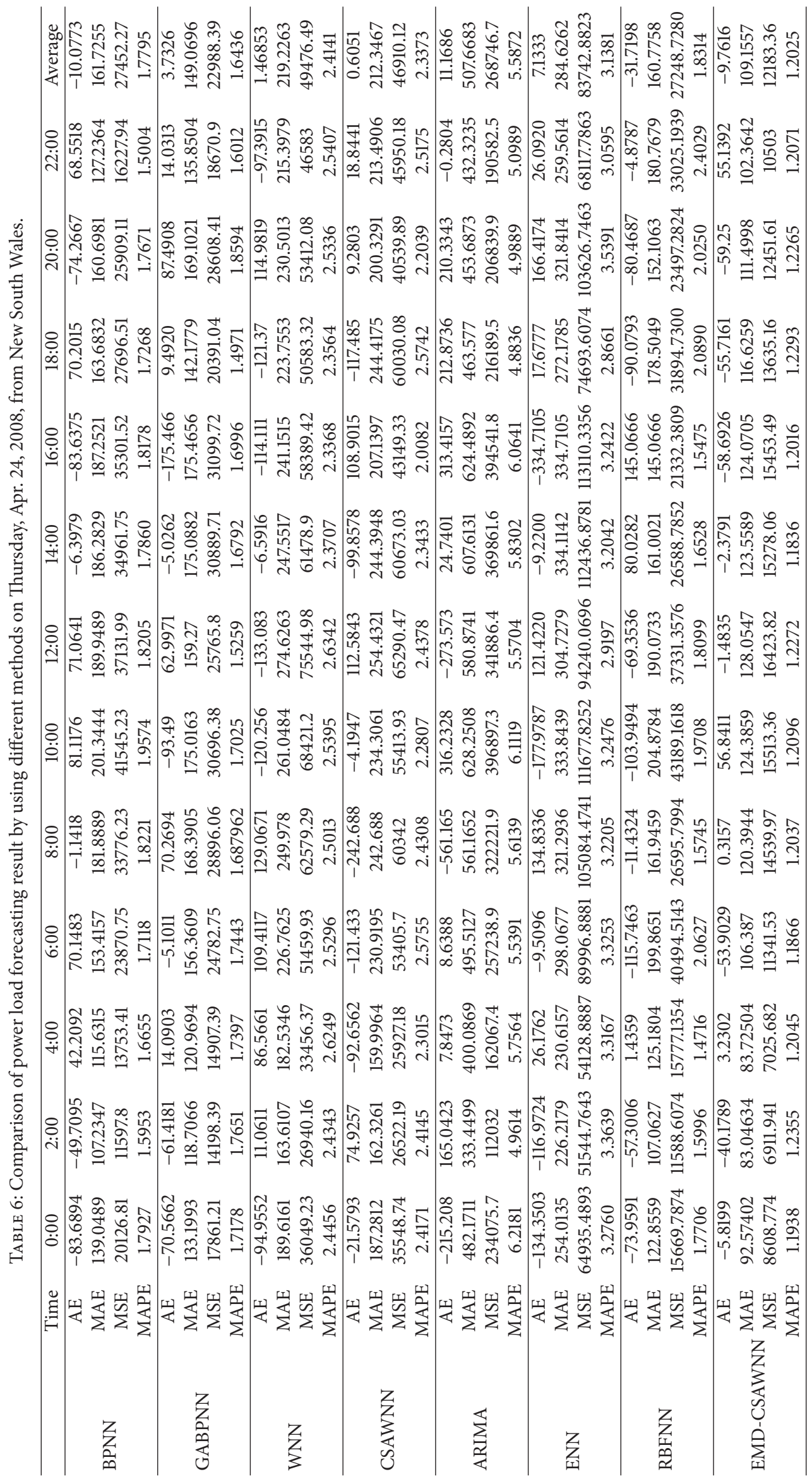




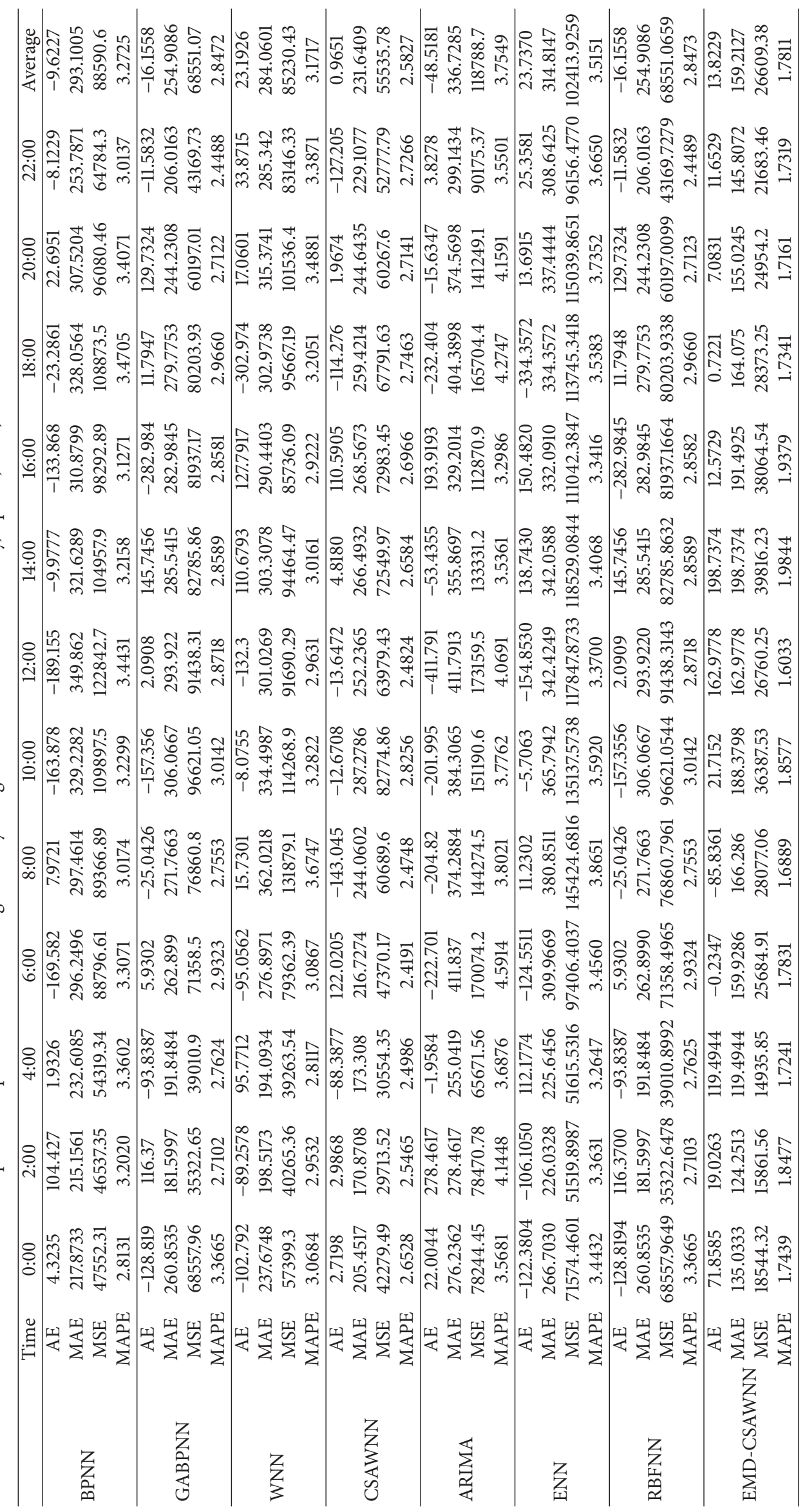




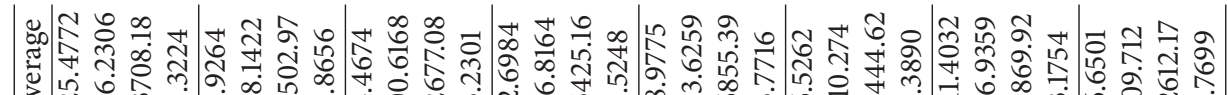

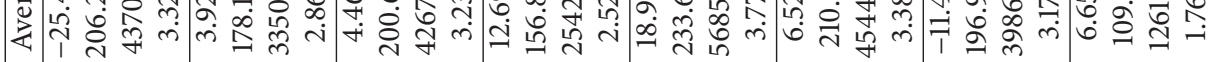

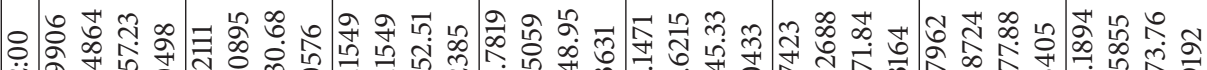

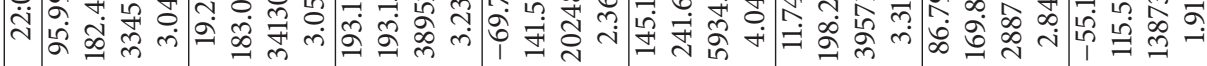

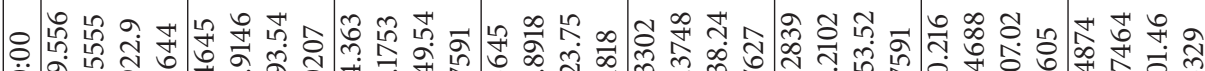

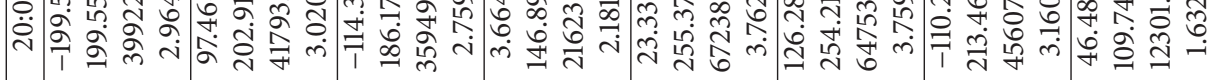

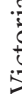

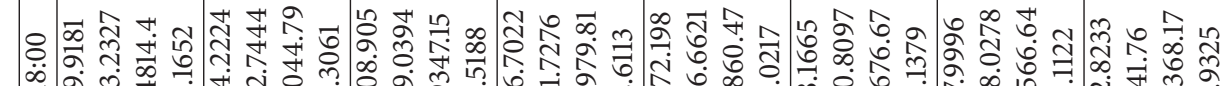
范

8

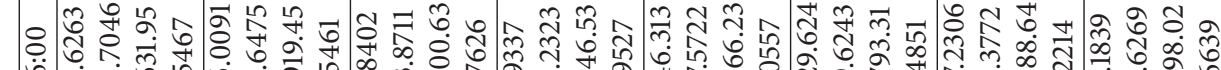

纨

○ी

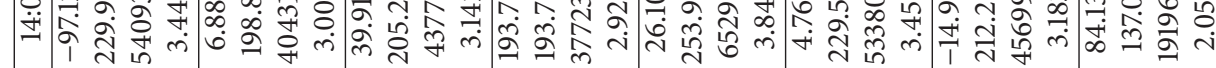

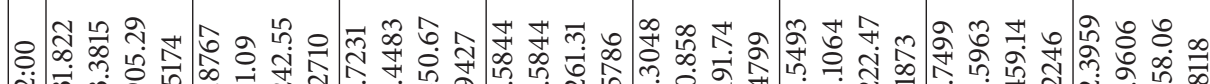
i

\&

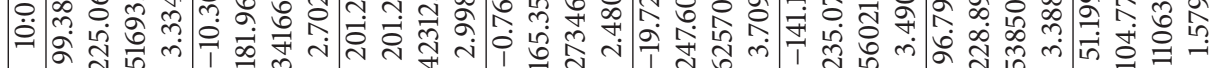

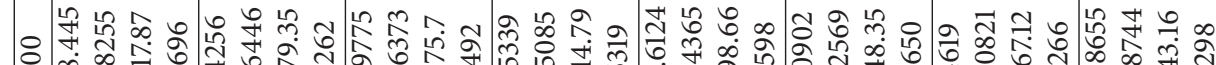

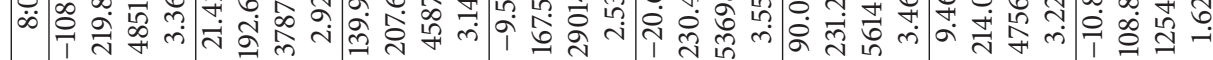

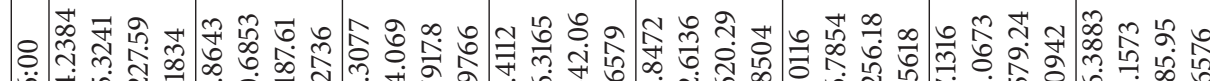
ن

蒫

\&乎 讨

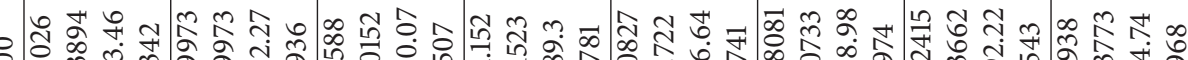

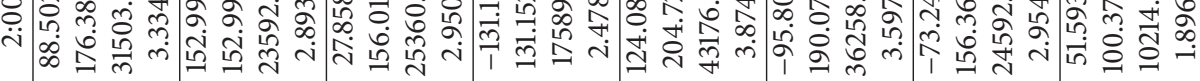

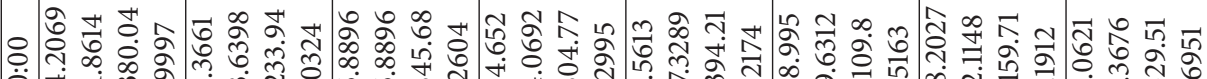

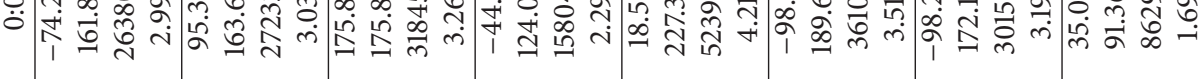
| 


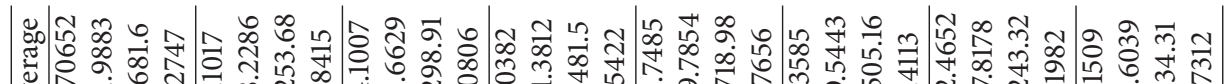

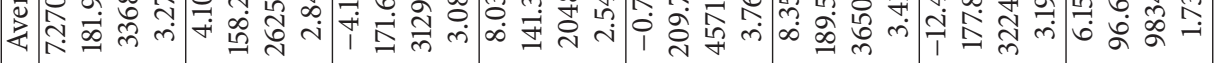

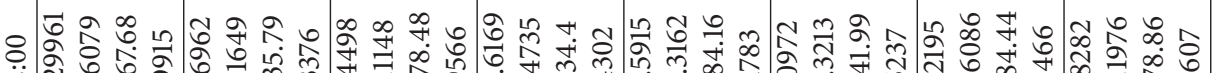

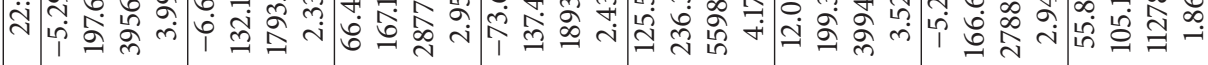

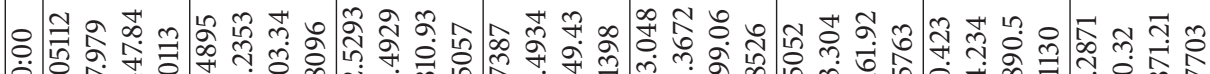
în

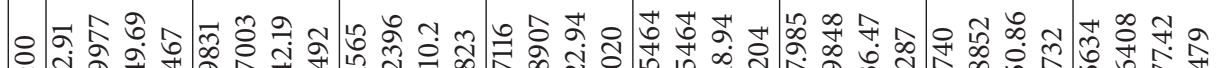

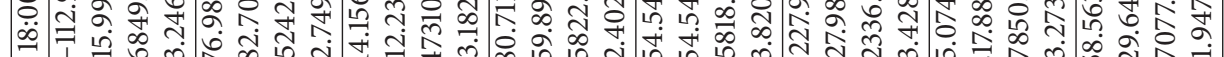

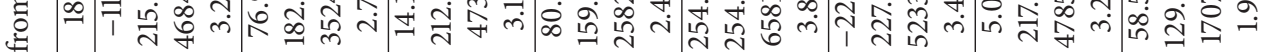

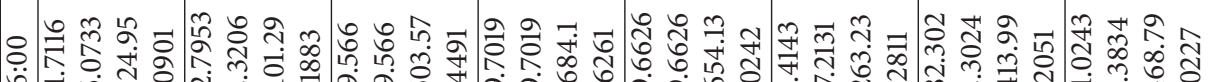

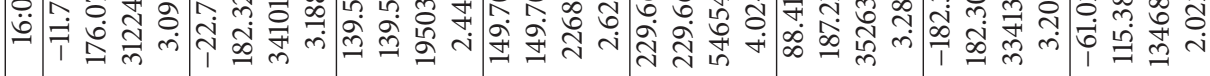

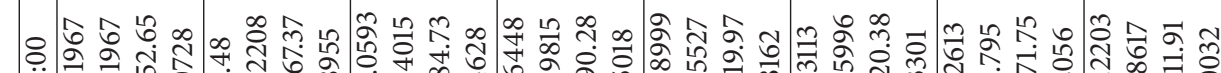

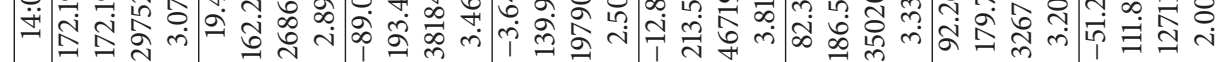

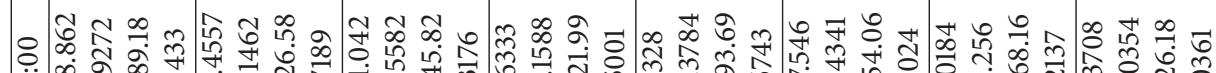

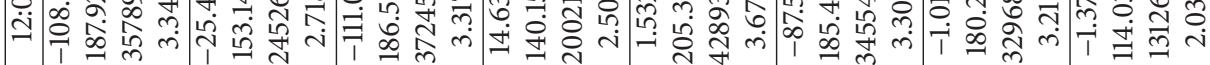

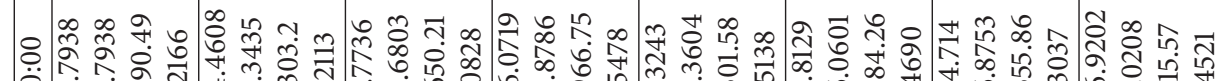

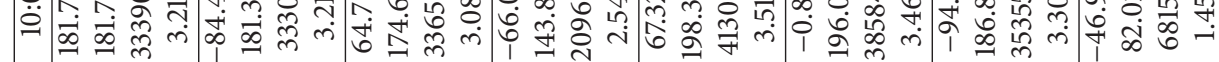

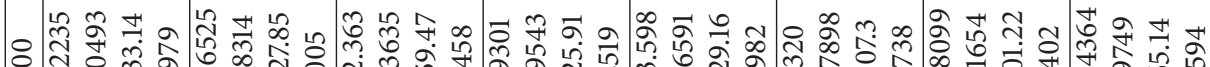
क人

○ी b|

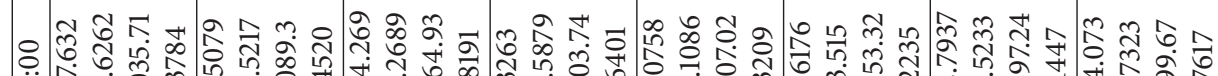

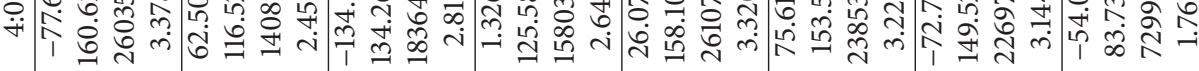

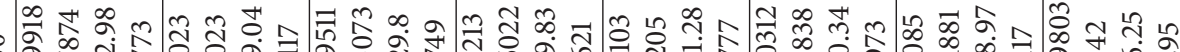

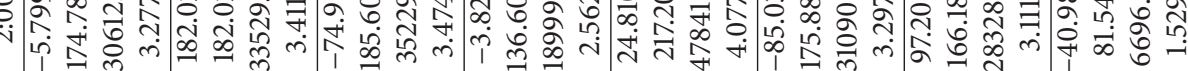

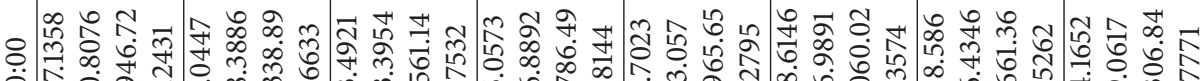
în

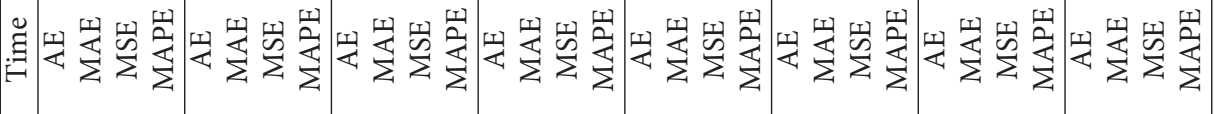

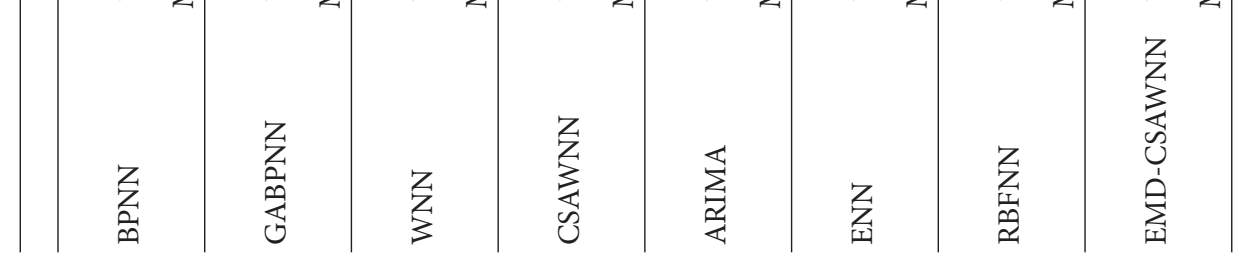




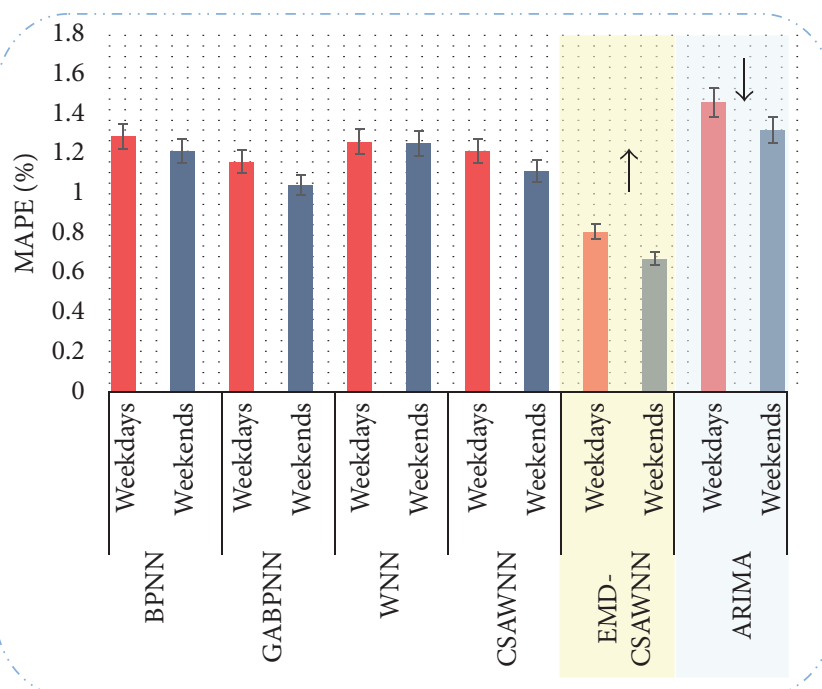

MAE

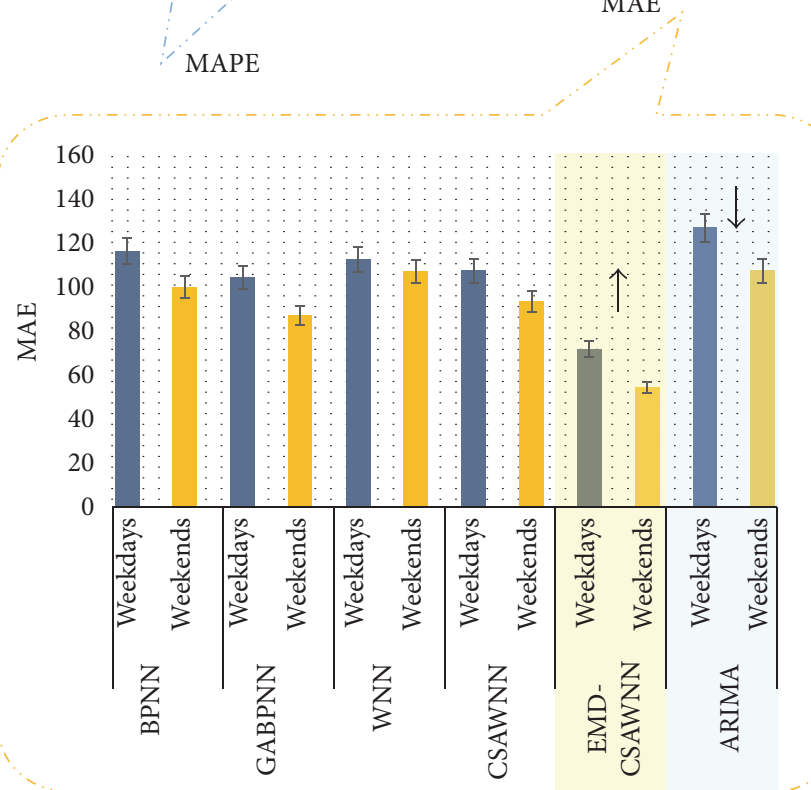

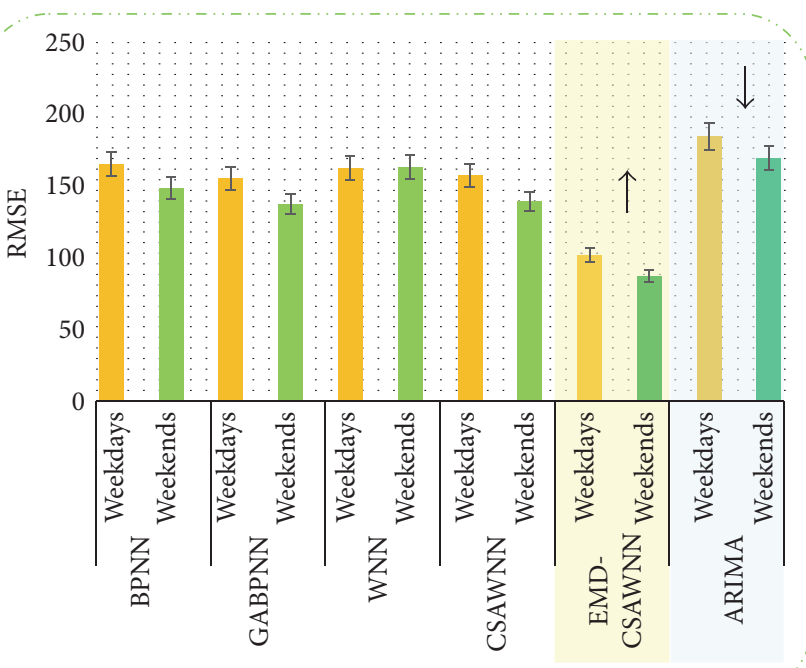

GRA

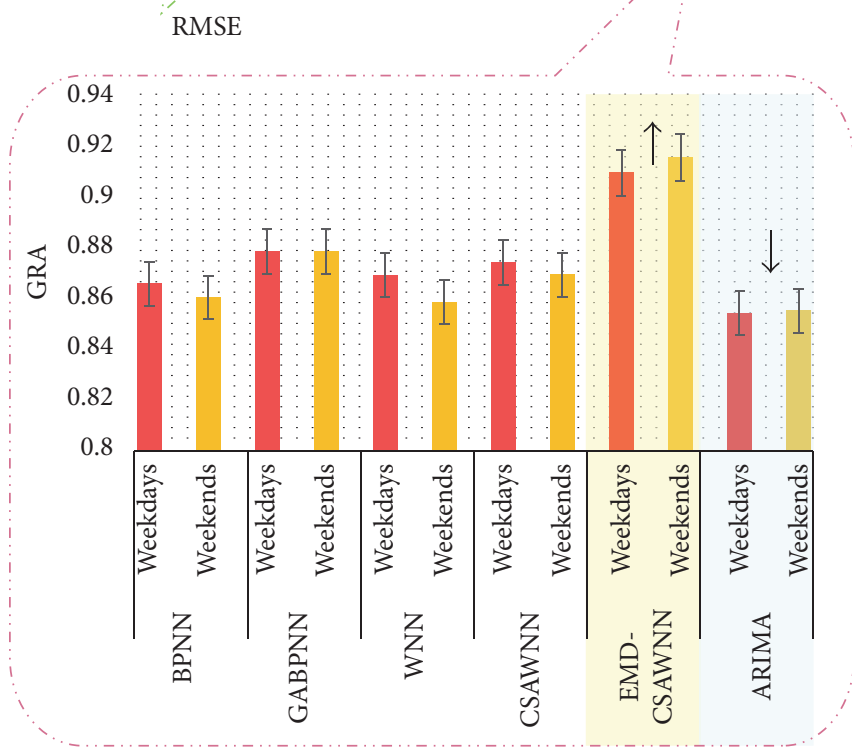

$\uparrow$ Best performance

$\downarrow$ Worst performance

FIGURE 8: The BPNN, GABPNN, WNN, CSAWNN, EMD-CSAWNN, and ARIMA forecasting results between weekdays and weekends.

further consideration; on that basis, this section will give a general discrete form of forecasting availability [51].

Definition 10. Let

$$
e_{i t}= \begin{cases}-1, & \frac{\left(x_{t}-x_{i t}\right)}{x_{t}}>1 \\ \frac{\left(x_{t}-x_{i t}\right)}{x_{t}}, & -1 \leq \frac{\left(x_{t}-x_{i t}\right)}{x_{t}}>1 \\ 1, & \frac{\left(x_{t}-x_{i t}\right)}{x_{t}}>1 .\end{cases}
$$

$e_{i t}$ denotes the relative forecasting error of $i$ th forecasting method at time $t, i=1,2, \ldots, m, t=1,2, \ldots, N$. The matrix $E=\left(e_{i t}\right)_{m \times N}$ is called the relative error matrix of the forecasting model.

Definition 11. $A_{i t}=1-\left|e_{i t}\right|$ is called the forecasting accuracy of $i$ th forecasting method at time $t, i=1,2, \ldots, m$, and $t=$ $1,2, \ldots, N$.

Definition 12. $m_{i}^{k}=\sum_{t=1}^{N} Q_{t} A_{i t}^{k}$ is called $k$ th-order forecasting availability unit of $i$ th forecasting method, $k$ is a positive integer, $i=1,2, \ldots, m,\left\{Q_{t}, t=1,2, \ldots, N\right\}$ is the discrete 


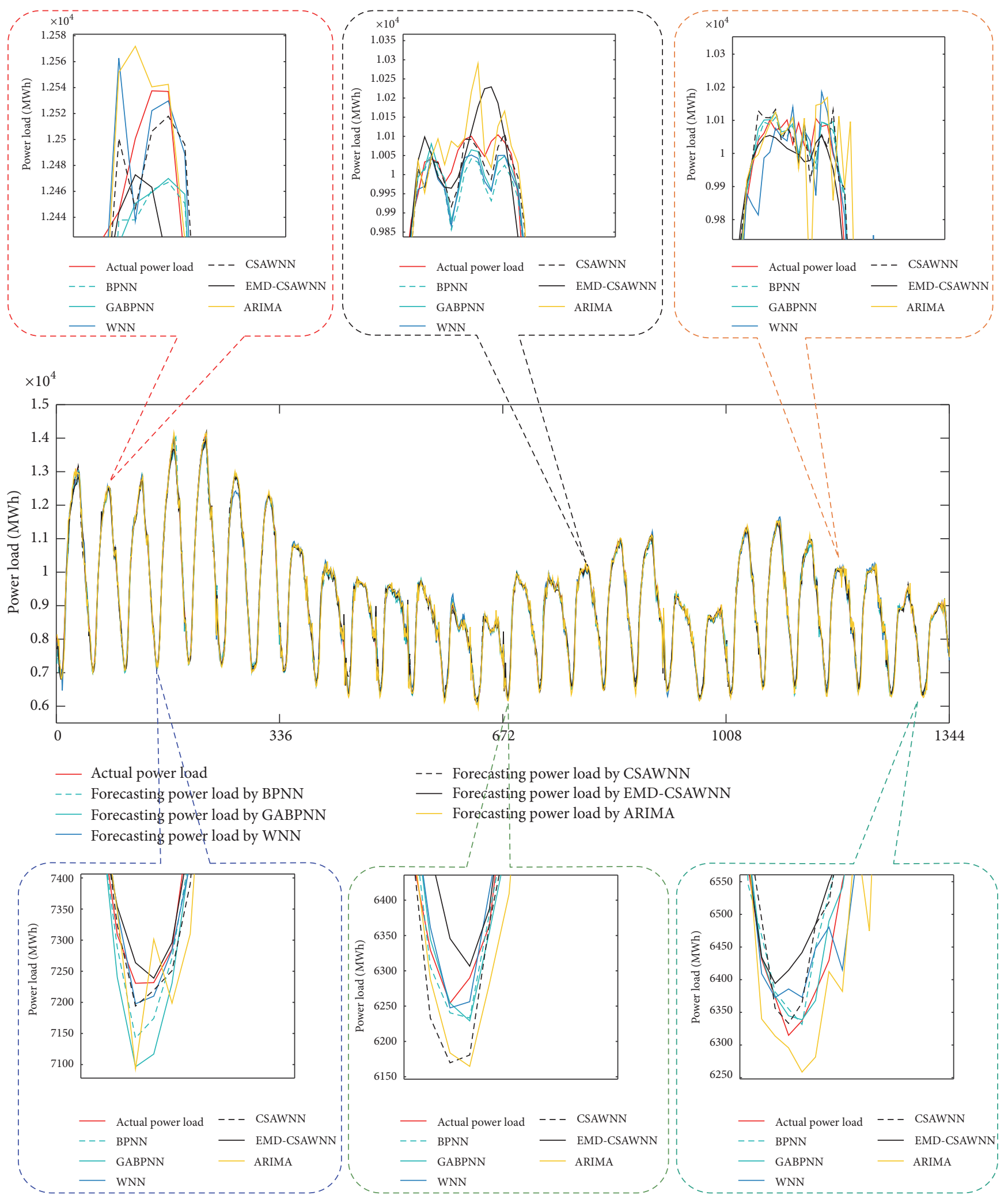

FIgURE 9: The forecasting results and actual values from February 2 to March 1. 

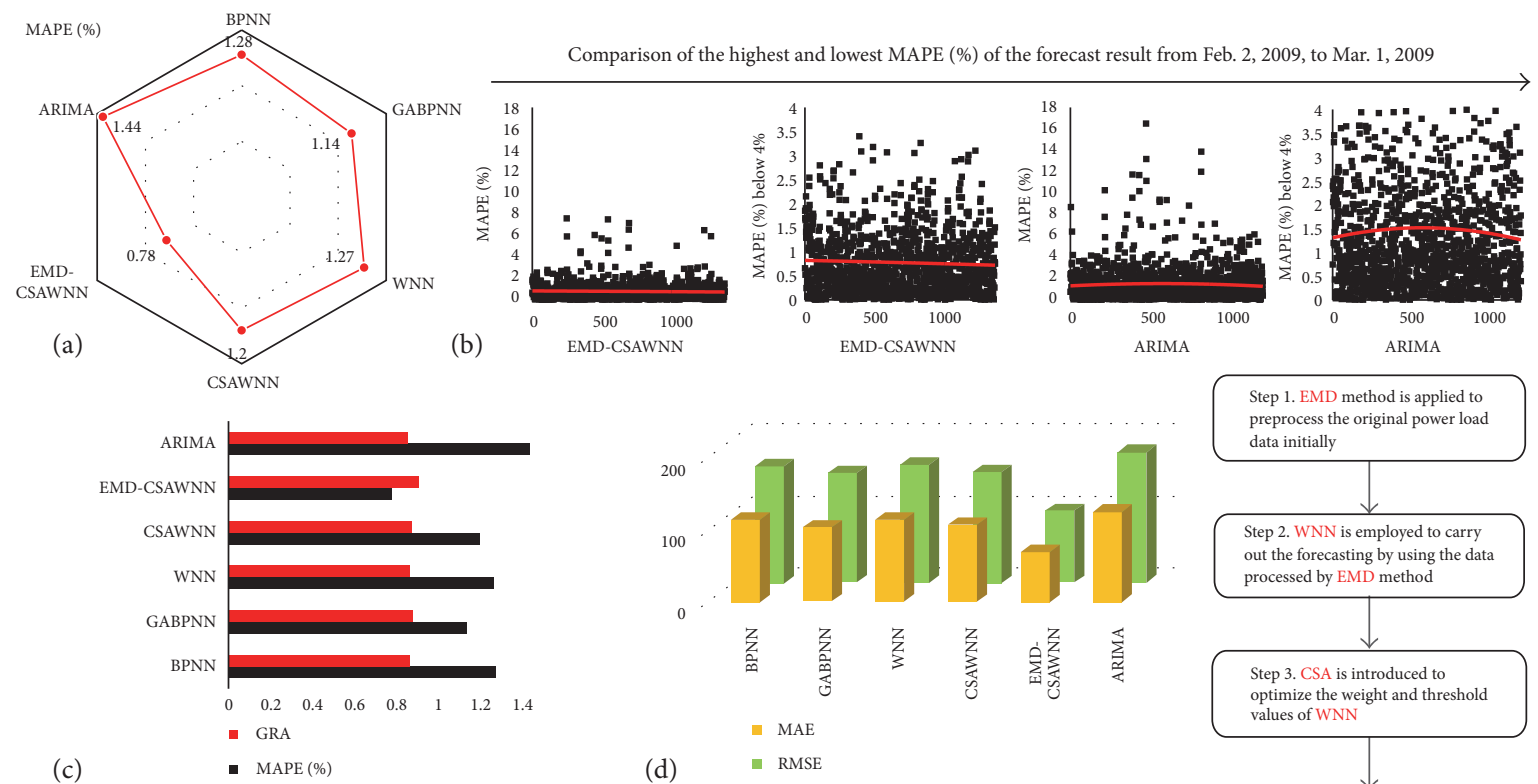

(c)

(d) $\quad$ RMSE
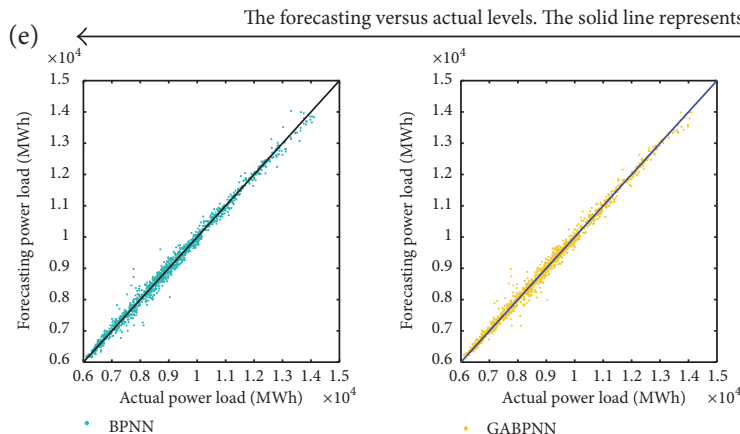

the perfect fit, and so forth, $y=x$
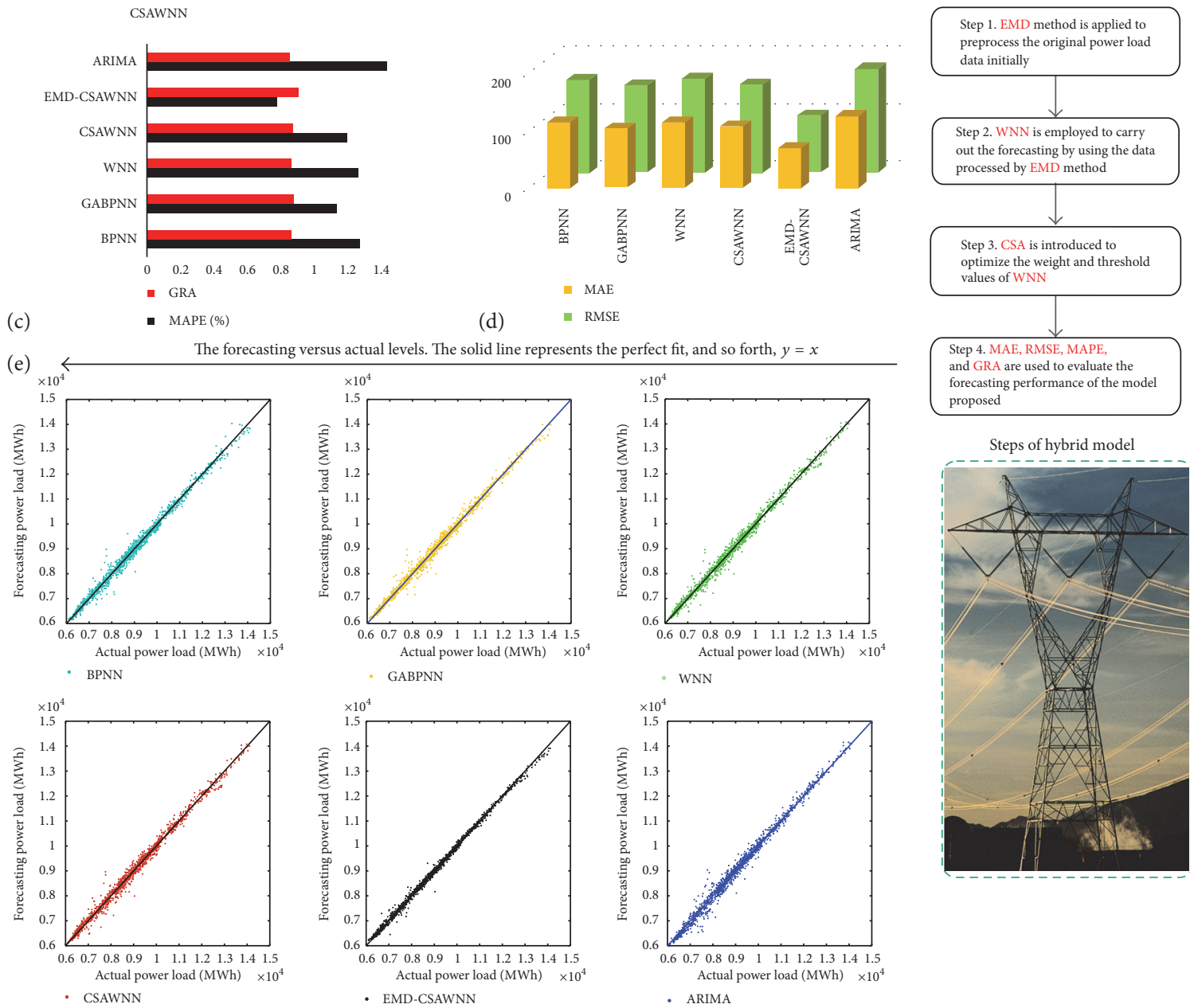
WNN
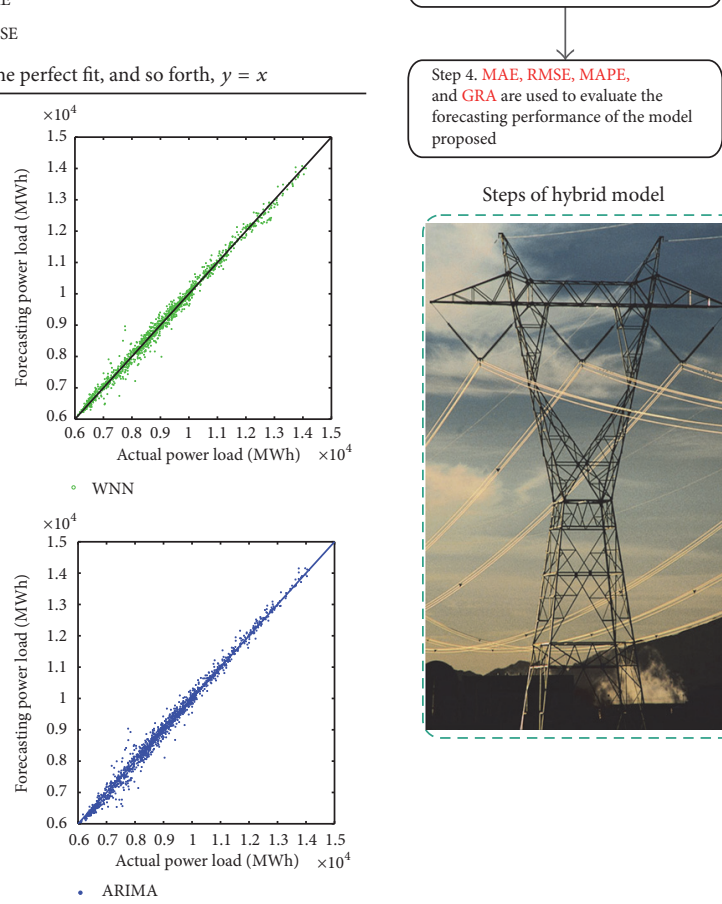
forecasting performance of the mode proposed

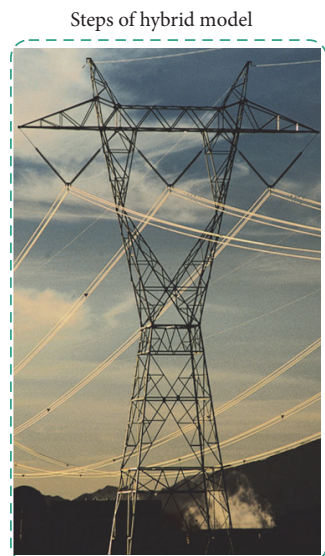

(f)

\begin{tabular}{lcccc}
\hline \multirow{2}{*}{ Model } & \multicolumn{4}{c}{ Evaluation from Feb. 2, 2009, to Mar. 1, 2009 } \\
& MAE & RMSE & MAPE (\%) & GRA \\
\hline BPNN & 113.2811 & 160.3951 & 1.28 & 0.8673 \\
GABPNN & 100.9882 & 150.0891 & 1.14 & 0.8811 \\
WNN & 112.7067 & 162.3033 & 1.27 & 0.8692 \\
CSAWNN & 105.0267 & 152.073 & 1.2 & 0.8756 \\
EMD-CSAWNN & 67.7484 & 97.6183 & 0.78 & 0.9127 \\
ARIMA & 123.2941 & 180.0273 & 1.44 & 0.8576 \\
\hline
\end{tabular}

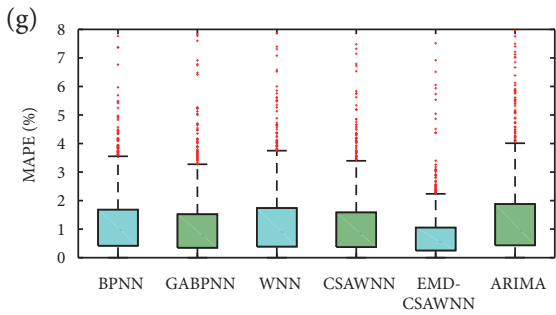

FIgURE 10: The comprehensive evaluation of forecasting models from February 2 to March 1. (a) The radar diagram of MAPE by using six different models. (b) Comparison of forecasting MAPE by using the EMD-CSAWNN and ARIMA models. The red line is the polynomial regression line. (c) The comparison of GRA and MAPE by using six different models. (d) The comparison of MAE and RMSE by using six different models. (e) The scatterplot of forecasting versus actual levels by using six different models. The solid line represents the perfect fit: that is, $y=x$. (f) Evaluation results of six different models. The red font is the best value of every evaluation index; the green font is the worst. (g) The comparison of the box plot by using six different models. The whiskers in the box plot indicate the primary range for the data, in which the lowest data are 1.5 times the interquartile range of the lower quartile and the highest data are 1.5 times the interquartile range of the upper quartile. The outliers, which are not included between the whiskers, are represented by the red crosshair. 

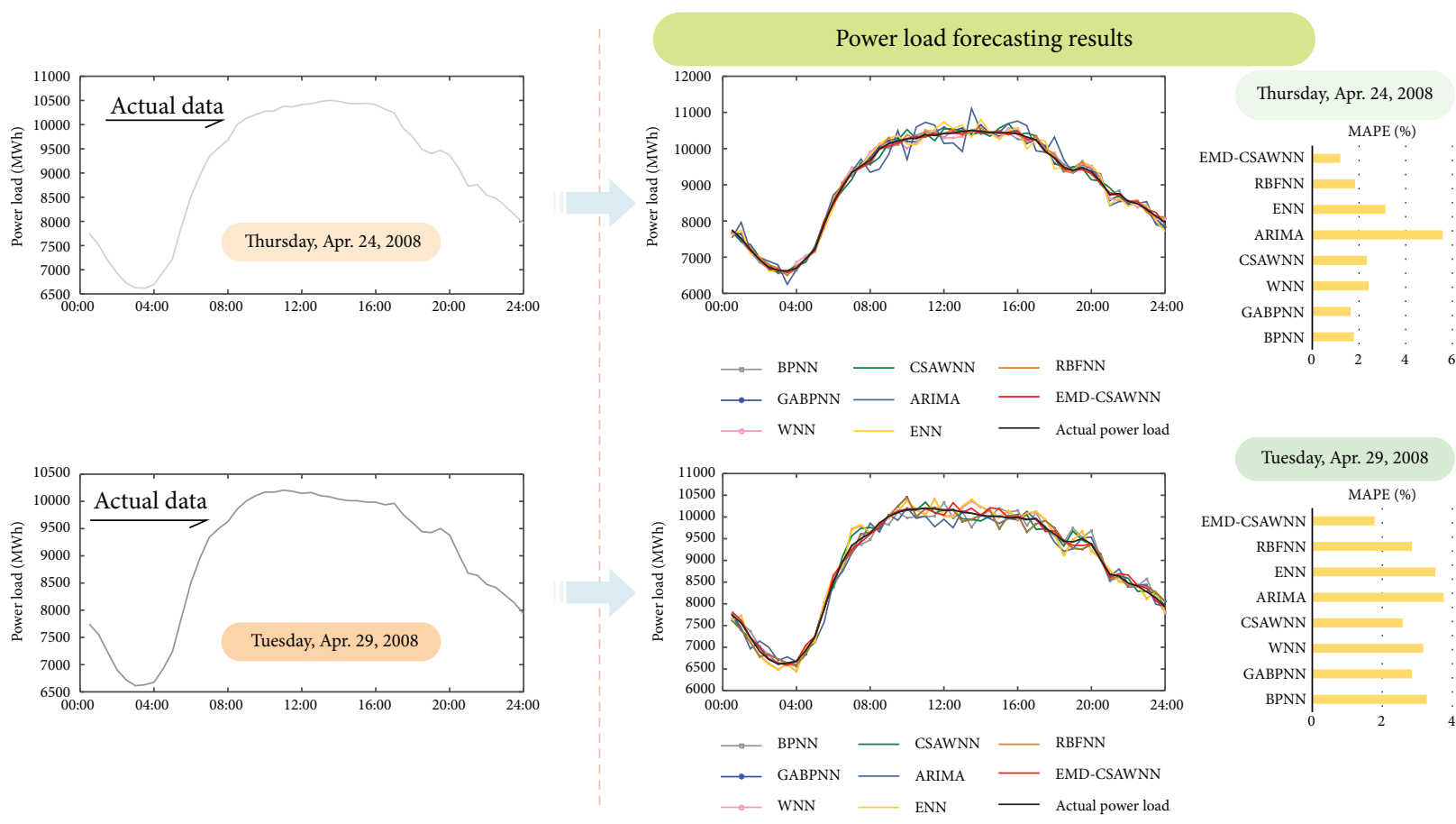

(a)

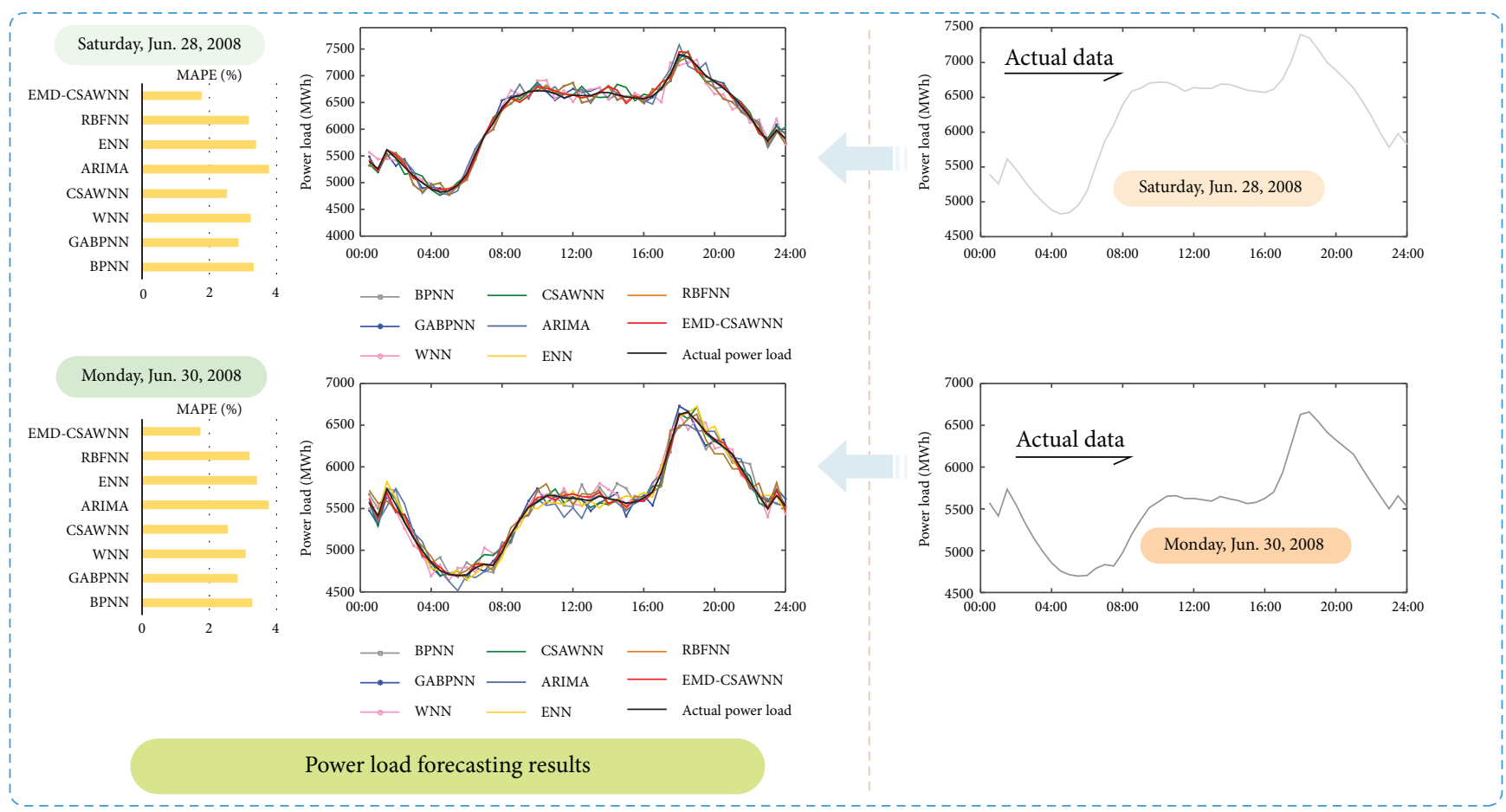

(b)

FIGURE 11: The comprehensive evaluation of forecasting models in Experiments 4 and 5. (a) Comparison of forecasting results by eight models on Thursday, April 24, 2008, and Tuesday, April 29, 2008, from New South Wales of Australia. (b) Comparison of forecasting results by eight models on Saturday, June 28, 2008, and Monday, June 30, 2008, from Victoria of Australia. 


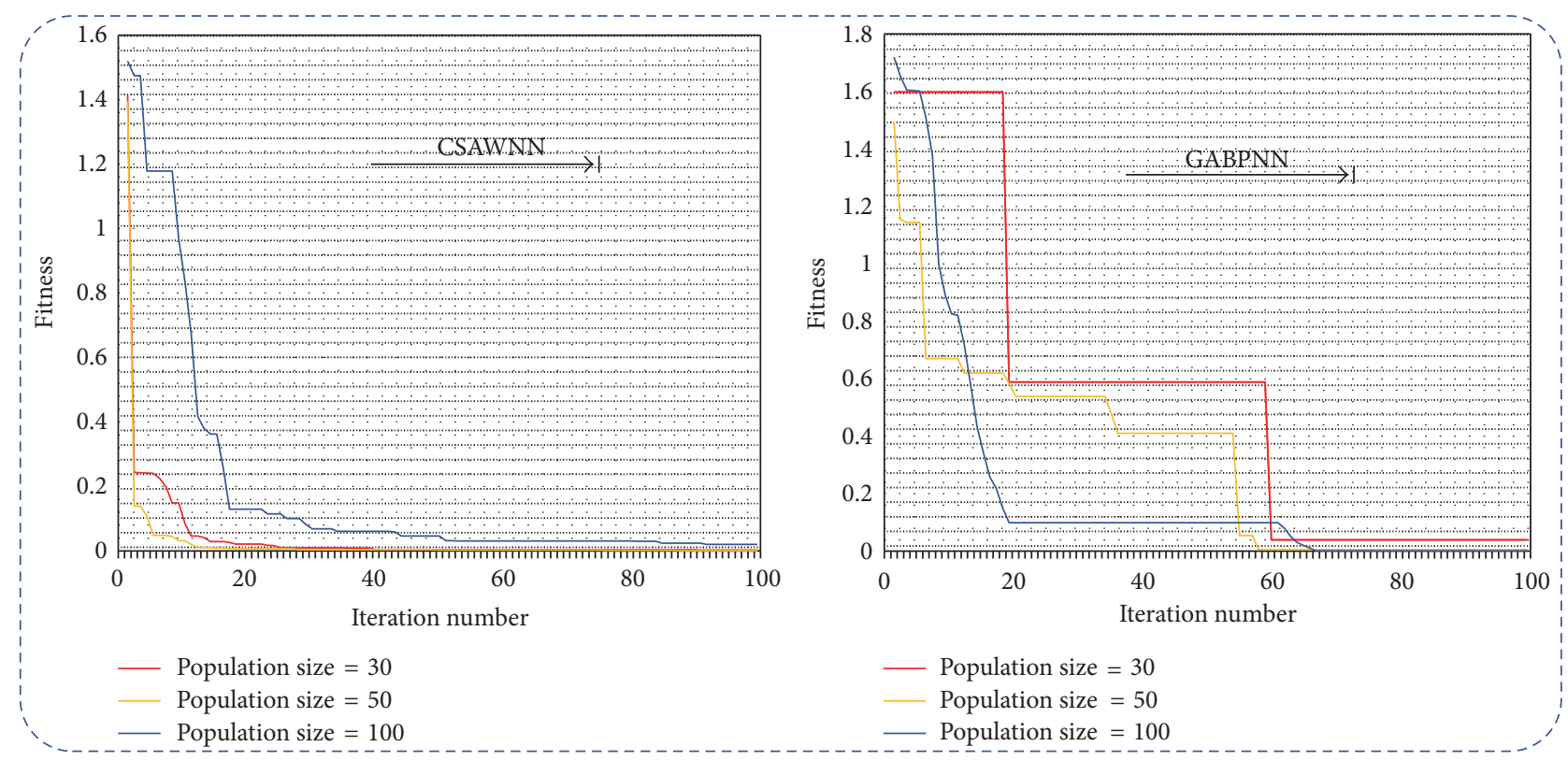

FIGURE 12: A convergence speed comparison of the fitness values between GA and CSA with different population sizes.

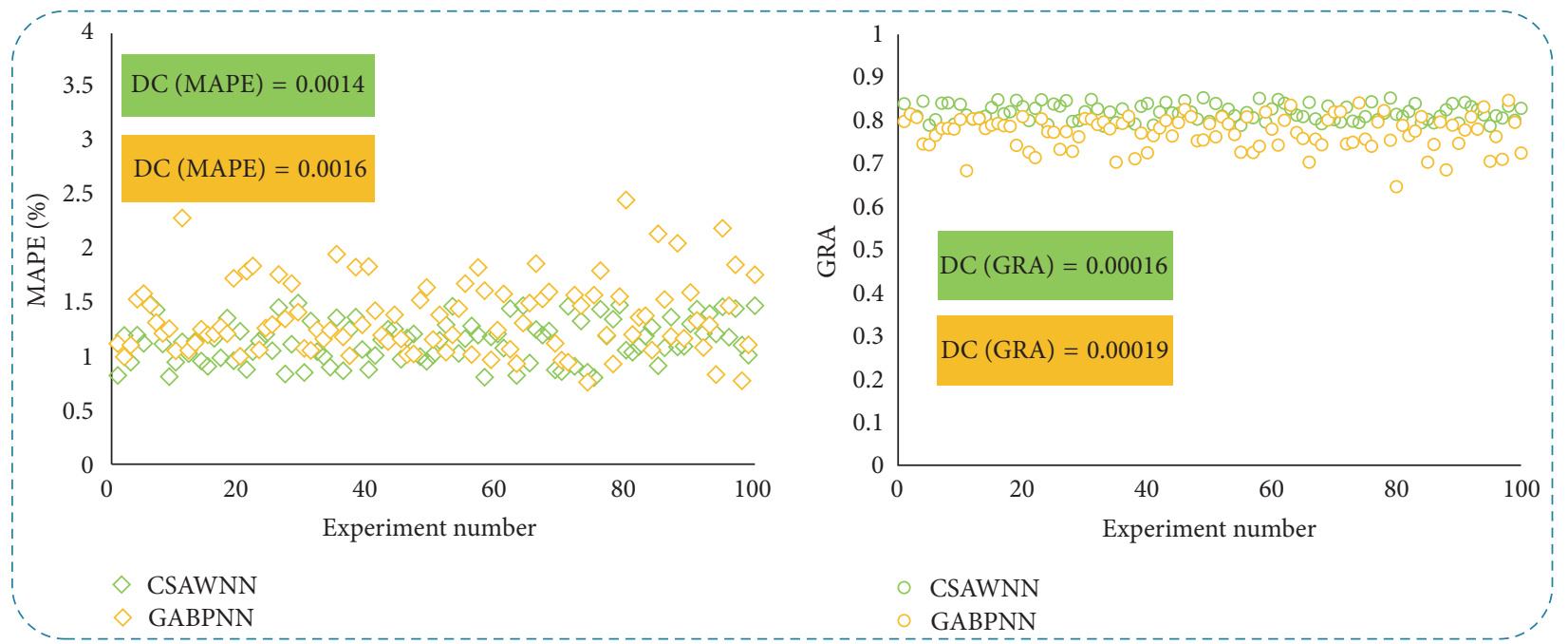

FIGURE 13: The DC (MAPE) and DC (GRA) of 100 experiments by comparison of GABPNN and CSAWNN.

probability distribution of $m$ types of methods at time $t$, and $\sum_{t=1}^{N} Q_{t}=1, Q_{t}>0$.

Especially if the priori information of the discrete probability distribution of $m$ types of methods is unknown, we define $Q_{t}=1 / N, t=1,2, \ldots, N$.

Definition 13. $m_{i}^{k}$ is called the $k$-order forecasting availability unit of $i$ th forecasting method, and $H$ is a continuous function of a certain $k$ unit. $H\left(m_{i}^{1}, m_{i}^{2}, \ldots, m_{i}^{k}\right)$ is called the $k$-order forecasting availability of $i$ th forecasting method.

Definition 14. When $H(x)=x$ is a continuous function of one variable, $H\left(m_{i}^{1}\right)=m_{i}^{1}$ is the 1-order forecasting availability of $i$ th forecasting method. When $H(x, y)=x\left(1-\sqrt{y-x^{2}}\right)$ is a continuous function of two variables, $H\left(m_{i}^{1}, m_{i}^{2}\right)=m_{i}^{1}(1-$ $\left.\sqrt{m_{i}^{2}-\left(m_{i}^{1}\right)^{2}}\right)$ is the 2-order forecasting availability of $i$ th forecasting method.

Especially if the first decimal of $H\left(m_{i}^{1}, m_{i}^{2}, \ldots, m_{i}^{k}\right)$ is the same, we define $H\left(m_{i}^{1}, m_{i}^{2}, \ldots, m_{i}^{k}\right)=\left\{10 H\left(m_{i}^{1}, m_{i}^{2}, \ldots\right.\right.$, $\left.\left.m_{i}^{k}\right)\right\}$, where $\{\cdot\}$ denotes the fractional part.

Definition 14 illustrates that the 1st-order forecasting availability is the expectation forecasting accuracy sequence. The 2nd-order forecasting availability is the difference between the expectation and standard deviation of the forecasting accuracy sequence. We use the forecasting availability to evaluate the power load forecasting results in this paper. Through Figure 14, we obtain that the 1st-order and 2nd-order 


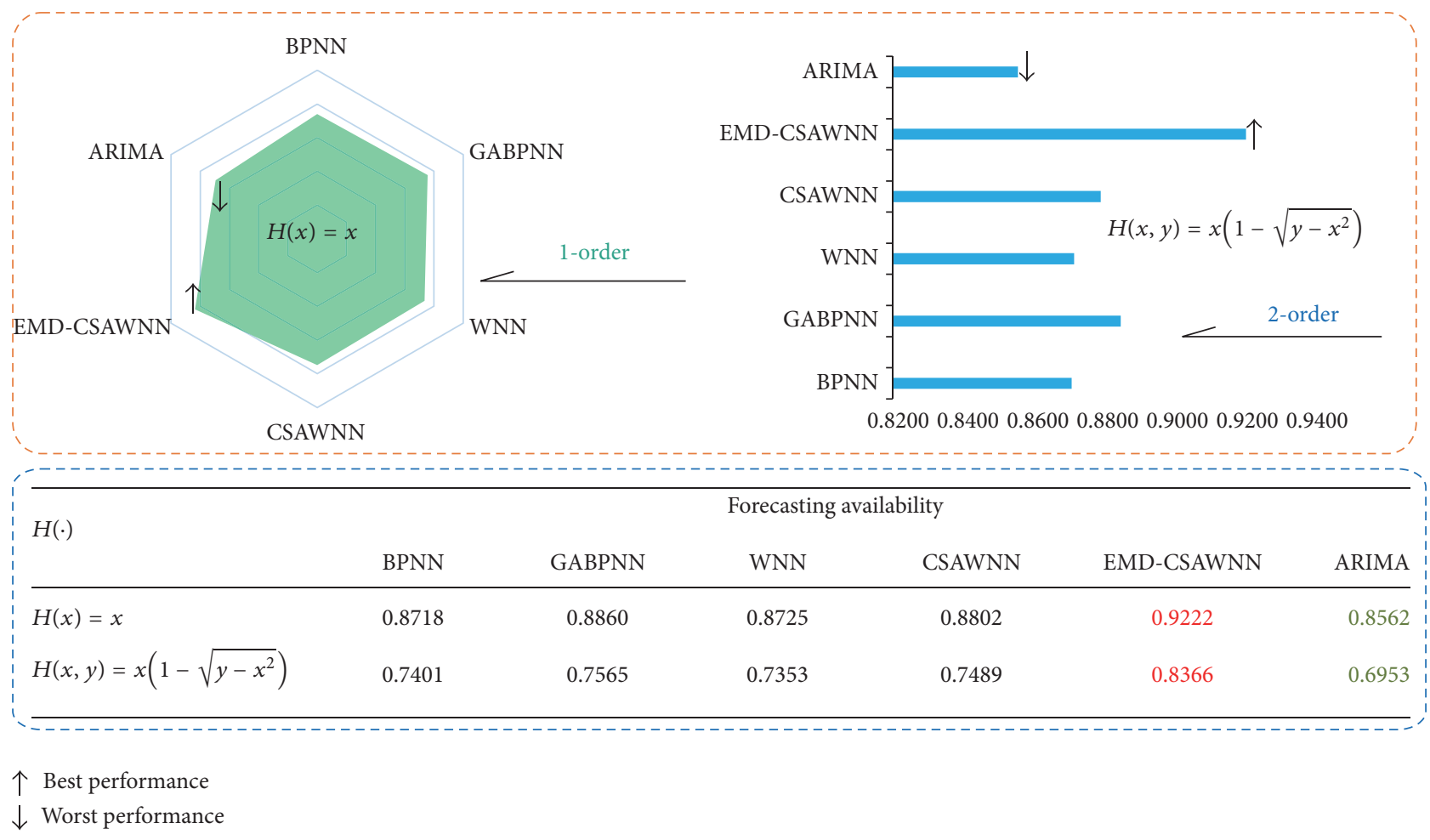

FIGURE 14: The forecasting availability of BPNN, GABPNN, WNN, CSAWNN, EMD-CSAWNN, and ARIMA.

forecasting availability offered by the hybrid model are 0.9222 and 0.8366 , respectively, which outperform those of the others; this evaluation result corresponds to the previous evaluation criterion. Thus, the hybrid model is a more valid model than the others.

\section{Conclusion}

The one-day-ahead power load forecasting is an extremely important problem in power load planning, secure operation, and energy expenditure economy. Assessment of the power load as accurately and quickly as possible is the primary objective in power load forecasting. However, power load is affected by various uncertain factors such as climate change and the social environment, which may lead to difficulty in obtaining accurate power load forecasts. The accuracy of traditional individual forecasting methods, which lack denoising, is not satisfactory for power load forecasting. Herein, a hybrid EMD-CSAWNN model for short-term power load forecasting is developed. The empirical model decomposition technique is applied to reduce the high-frequency items. On the basis that WNN can handle the data with nonlinear features, the ensemble forecasting method is adopted to overcome the uncertainty of the outcomes that can be attributed to the randomness of the initialization of the single WNN. Moreover, we use the CSA to optimize the parameter in the ensemble forecasting model. Experimental studies of power load forecasting in NSW demonstrate that the hybrid model has higher precision than conventional forecasting models. The proposed EMD-CSAWNN model can provide efficient computation and satisfactory forecasting accuracy for this type of data. Therefore, the developed hybrid approach is suggested for broad application in power load forecasts or even other fields such as wind speed and traffic flow forecasts.

\section{Abbreviations}

BPNN: Back Propagation Neural Network

WNN: Wavelet Neural Network

GA: $\quad$ Genetic Algorithm

CSA: Cuckoo Search Algorithm Back Propagation Neural

GABPNN: Network Optimized by Genetic Algorithm

CSAWNN: Wavelet Neural Network Optimized by Cuckoo Search Algorithm

AR: $\quad$ Autoregressive model

MA: $\quad$ Moving average model

ARIMA: Autoregressive integrated moving average model

IMF: Intrinsic mode function

ANN: $\quad$ Artificial Neural Network

PSO: $\quad$ Particle swarm optimization algorithm

ACO: Ant colony optimization algorithm

SAO: $\quad$ Simulated annealing optimization algorithm

MAE: Mean absolute error

RMSE: Root mean square error

MAPE: Mean absolute percentage error

GRA: Grey relational analysis

q: $\quad$ The number of sample data points used to build the NN model 


\begin{tabular}{|c|c|}
\hline$p:$ & $\begin{array}{l}\text { The number of data points to be forecasted in } \\
\text { the NN model }\end{array}$ \\
\hline$\omega:$ & $\begin{array}{l}\text { The connection weights between the neurons } \\
\text { of the input layer, hidden layer, and output } \\
\text { layer, with values belonging to }[-1,1]\end{array}$ \\
\hline$f(\cdot):$ & The excitation function of the hidden layer \\
\hline$\eta:$ & $\begin{array}{l}\text { The learning rate of the NN, which is used to } \\
\text { adjust the weights and thresholds of the NN }\end{array}$ \\
\hline iter $_{\max }$ : & The maximum number of iterations \\
\hline$P:$ & $\begin{array}{l}\text { The population size of the initial population } \\
\text { space }\end{array}$ \\
\hline$b:$ & A random number belonging to $[0,1]$ \\
\hline$r:$ & A random number belonging to $[0,1]$ \\
\hline$w_{\max }^{\text {iter }}, w_{\min }^{\text {iter }}:$ & $\begin{array}{l}\text { The higher and lower bounds of the value of } \\
\text { the gene } w_{i j}^{\text {iter }}\end{array}$ \\
\hline$G_{\max }:$ & The maximum number of generations \\
\hline$P_{\alpha}:$ & $\begin{array}{l}\text { The possibility of finding an exotic egg by the } \\
\text { nest master }\end{array}$ \\
\hline$X_{*}^{\text {iter }}:$ & $\begin{array}{l}\text { The location of the optimum nest in } \\
\text { generation iter }\end{array}$ \\
\hline$\chi_{j}^{\text {iter }}, \chi_{k}^{\text {iter }}:$ & Two random numbers in generation iter. \\
\hline
\end{tabular}

\section{Competing Interests}

The authors declare that they have no competing interests.

\section{Acknowledgments}

The National Natural Science Foundation of China supported this work \{Grant no. 71171102\}.

\section{References}

[1] S. Kouhi, F. Keynia, and S. Najafi Ravadanegh, "A new shortterm load forecast method based on neuro-evolutionary algorithm and chaotic feature selection," International Journal of Electrical Power and Energy Systems, vol. 62, pp. 862-867, 2014.

[2] N. Amjady, "Short-term hourly load forecasting using timeseries modeling with peak load estimation capability," IEEE Transactions on Power Systems, vol. 16, no. 3, pp. 498-505, 2001.

[3] Y. Zhang and G. Luo, "Short term power load prediction with knowledge transfer," Information Systems, vol. 53, pp. 161-169, 2015.

[4] N. An, W. Zhao, J. Wang, D. Shang, and E. Zhao, "Using multi-output feedforward neural network with empirical mode decomposition based signal filtering for electricity demand forecasting," Energy, vol. 49, no. 1, pp. 279-288, 2013.

[5] I. Moghram and S. Rahman, "Analysis and evaluation of five short-term load forecasting techniques," IEEE Transactions on Power Systems, vol. 4, no. 4, pp. 1484-1491, 1989.

[6] M. T. Hagan and S. M. Behr, "The time series approach to short term load forecasting," IEEE Transactions on Power Systems, vol. 2, no. 3, pp. 785-791, 1987.

[7] P. G. Zhang, "Time series forecasting using a hybrid ARIMA and neural network model," Neurocomputing, vol. 50, pp. 159175, 2003.

[8] V. Dordonnat, S. J. Koopman, M. Ooms, A. Dessertaine, and J. Collet, "An hourly periodic state space model for modelling French national electricity load," International Journal of Forecasting, vol. 24, no. 4, pp. 566-587, 2008.
[9] W. R. Christiaanse, "Short-term load forecasting using general exponential smoothing," IEEE Transactions on Power Apparatus and Systems, vol. 90, no. 2, pp. 900-911, 1971.

[10] W.-C. Hong, "Hybrid evolutionary algorithms in a SVR-based electric load forecasting model," International Journal of Electrical Power \& Energy Systems, vol. 31, no. 7-8, pp. 409-417, 2009.

[11] V. H. Hinojosa and A. Hoese, "Short-term load forecasting using fuzzy inductive reasoning and evolutionary algorithms," IEEE Transactions on Power Systems, vol. 25, no. 1, pp. 565-574, 2010.

[12] P. K. Dash, A. C. Liew, S. Rahman, and G. Ramakrishna, "Building a fuzzy expert system for electric load forecasting using a hybrid neural network," Expert Systems with Applications, vol. 9, no. 3, pp. 407-421, 1995.

[13] S. Rahman and O. Hazim, "Load forecasting for multiple sites: development of an expert system-based technique," Electric Power Systems Research, vol. 39, no. 3, pp. 161-169, 1996.

[14] D. K. Chaturvedi, A. P. Sinha, and O. P. Malik, "Short term load forecast using fuzzy logic and wavelet transform integrated generalized neural network," International Journal of Electrical Power and Energy Systems, vol. 67, pp. 230-237, 2015.

[15] S. Kouhi and F. Keynia, "A new cascade NN based method to short-term load forecast in deregulated electricity market," Energy Conversion and Management, vol. 71, pp. 76-83, 2013.

[16] P. Li, Y. Li, Q. Xiong, Y. Chai, and Y. Zhang, "Application of a hybrid quantized Elman neural network in short-term load forecasting," International Journal of Electrical Power \& Energy Systems, vol. 55, pp. 749-759, 2014.

[17] H. S. Hippert, C. E. Pedreira, and R. C. Souza, "Neural networks for short-term load forecasting: a review and evaluation," IEEE Transactions on Power Systems, vol. 16, no. 1, pp. 44-55, 2001.

[18] R. Mamlook, O. Badran, and E. Abdulhadi, "A fuzzy inference model for short-term load forecasting," Energy Policy, vol. 37, no. 4, pp. 1239-1248, 2009.

[19] M. Hanmandlu and B. K. Chauhan, "Load forecasting using hybrid models," IEEE Transactions on Power Systems, vol. 26, no. 1, pp. 20-29, 2011.

[20] M. Q. Raza and Z. Baharudin, "A review on short term load forecasting using hybrid neural network techniques," in Proceedings of the International Conference on Power and Energy (PECon '12), pp. 846-851, IEEE, Kota Kinabalu, Malaysia, December 2012.

[21] M. El-Telbany and F. El-Karmi, "Short-term forecasting of Jordanian electricity demand using particle swarm optimization," Electric Power Systems Research, vol. 78, no. 3, pp. 425-433, 2008.

[22] A. B. Nutt, R. C. Lenz Jr., H. W. Lanford, and M. J. Cleary, "Data sources for trend extrapolation in technological forecasting," Long Range Planning, vol. 9, no. 1, pp. 72-76, 1976.

[23] M. M. Tripathi, K. G. Upadhyay, and S. N. Singh, "Short-term load forecasting using generalized regression and probabilistic neural networks in the electricity market," The Electricity Journal, vol. 21, no. 9, pp. 24-34, 2008.

[24] A. D. Papalexopoulos and T. C. Hesterberg, "A regression-based approach to short-term system load forecasting," IEEE Transactions on Power Systems, vol. 5, no. 4, pp. 1535-1547, 1990.

[25] N. Ding, Y. Bésanger, and F. Wurtz, "Next-day MV/LV substation load forecaster using time series method," Electric Power Systems Research, vol. 119, pp. 345-354, 2015.

[26] O. Valenzuela, I. Rojas, F. Rojas et al., "Hybridization of intelligent techniques and ARIMA models for time series prediction," Fuzzy Sets and Systems, vol. 159, no. 7, pp. 821-845, 2008. 
[27] H. Nie, G. Liu, X. Liu, and Y. Wang, "Hybrid of ARIMA and SVMs for short-term load forecasting," Energy Procedia, vol. 16, pp. 1455-1460, 2012.

[28] T. Yalcinoz and U. Eminoglu, "Short term and medium term power distribution load forecasting by neural networks," Energy Conversion and Management, vol. 46, no. 9-10, pp. 1393-1405, 2005.

[29] C. J. Bennett, R. A. Stewart, and J. W. Lu, "Forecasting low voltage distribution network demand profiles using a pattern recognition based expert system," Energy, vol. 67, pp. 200-212, 2014.

[30] H. S. Chen and W. C. Chang, "A study of optimal grey model GM $(1,1)$," Journal of the Chinese Grey System Association, vol. 1, no. 2, pp. 141-145, 1998.

[31] L. Xiao, J. Wang, X. Yang, and L. Xiao, "A hybrid model based on data preprocessing for electrical power forecasting," International Journal of Electrical Power and Energy Systems, vol. 64, pp. 311-327, 2015.

[32] J. X. Che and J. Z. Wang, "Short-term load forecasting using a kernel-based support vector regression combination model," Applied Energy, vol. 132, pp. 602-609, 2014.

[33] N. Liu, Q. Tang, J. Zhang, W. Fan, and J. Liu, "A hybrid forecasting model with parameter optimization for short-term load forecasting of micro-grids," Applied Energy, vol. 129, pp. 336345, 2014.

[34] S. Bahrami, R.-A. Hooshmand, and M. Parastegari, "Short term electric load forecasting by wavelet transform and grey model improved by PSO (particle swarm optimization) algorithm," Energy, vol. 72, pp. 434-442, 2014.

[35] L. Karthikeyan and D. N. Kumar, "Predictability of nonstationary time series using wavelet and EMD based ARMA models," Journal of Hydrology, vol. 502, pp. 103-119, 2013.

[36] G. K. Sharma, A. Kumar, T. Jayakumar, B. P. Rao, and N. Mariyappa, "Ensemble Empirical Mode Decomposition based methodology for ultrasonic testing of coarse grain austenitic stainless steels," Ultrasonics, vol. 57, pp. 167-178, 2015.

[37] A. Baliyan, K. Gaurav, and S. K. Mishra, "A review of short term load forecasting using artificial neural network models," Procedia Computer Science, vol. 48, pp. 121-125, 2015.

[38] R. Chao, [Ph.D. thesis], Lanzhou University, 2013.

[39] N. B. Andersen, "Real Paley-Wiener theorems and Roe's theorem associated with the Opdam-Cherednik transform," Journal of Mathematical Analysis and Applications, vol. 427, no. 1, pp. 47-59, 2015.

[40] K. Liu, W. Y. Guo, X. L. Shen, and Z. F. Tan, "Research on the forecast model of electricity power industry loan based on GABP neural network," Energy Procedia, vol. 4, pp. 1918-1924, 2012.

[41] Q. Zhang and A. Benveniste, "Wavelet networks," IEEE Transactions on Neural Networks, vol. 3, no. 6, pp. 889-898, 1992.

[42] Y. Lu, N. Zeng, Y. Liu, and N. Zhang, "A hybrid wavelet neural network and switching particle swarm optimization algorithm for face direction recognition," Neurocomputing, vol. 155, pp. 219-224, 2015.

[43] N. M. Nawi, A. Khan, and M. Z. Rehman, "A new back-propagation neural network optimized with cuckoo search algorithm," in Computational Science and Its Applications_ICCSA 2013: 13th International Conference, Ho Chi Minh City, Vietnam, June 24-27, 2013, Proceedings, Part I, vol. 7971 of Lecture Notes in Computer Science, pp. 413-426, Springer, Berlin, Germany, 2013.
[44] A. H. Gandomi, X.-S. Yang, and A. H. Alavi, "Cuckoo search algorithm: a metaheuristic approach to solve structural optimization problems," Engineering with Computers, vol. 29, no. 1, pp. 17-35, 2013.

[45] F. Yu and X. Xu, "A short-term load forecasting model of natural gas based on optimized genetic algorithm and improved BP neural network," Applied Energy, vol. 134, pp. 102-113, 2014.

[46] K. Liu, W. Guo, X. Shen, and Z. Tan, "Research on the gforecast model of electricity power industry loan based on GA-BP neural network," Energy Procedia, vol. 14, pp. 1918-1924, 2012.

[47] M. Smith, "Modeling and short-term forecasting of new south wales electricity system load," Journal of Business and Economic Statistics, vol. 18, no. 4, pp. 465-478, 2000.

[48] S. Qin, F. Liu, J. Wang, and B. Sun, "Analysis and forecasting of the particulate matter (PM) concentration levels over four major cities of China using hybrid models," Atmospheric Environment, vol. 98, pp. 665-675, 2014.

[49] D. Bunn and E. D. Farmer, Comparative Models for Electrical Load Forecasting, John Wiley \& Sons, Chichester, UK, 1985.

[50] L. Liu, Q. Wang, J. Wang, and M. Liu, "A rolling grey model optimized by particle swarm optimization in economic prediction," Computational Intelligence, 2014.

[51] H. Y. Chen and D. P. Hou, "Research on superior combination forecasting models based on forecasting effective measure," Journal of University of Science and Technology of China, vol. 32, no. 2, pp. 172-180, 2002. 


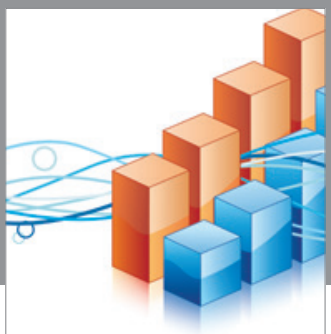

Advances in

Operations Research

vatem alat4

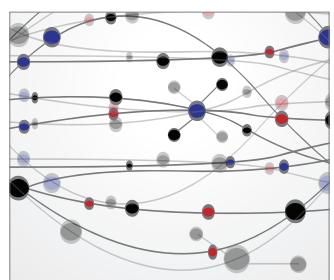

\section{The Scientific} World Journal
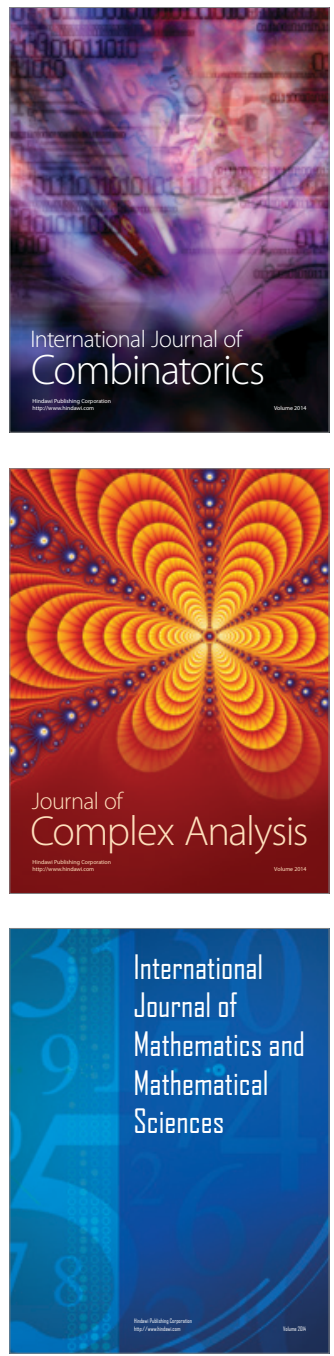
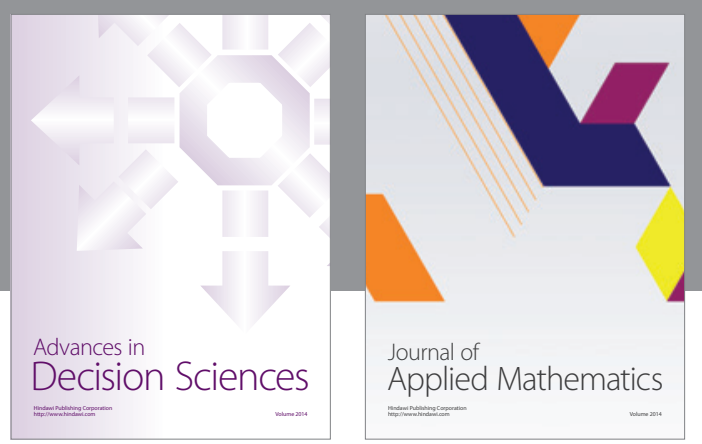

Algebra

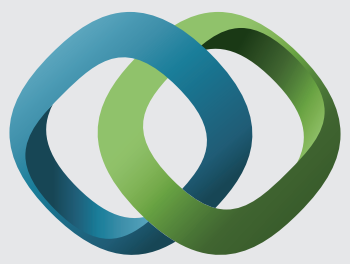

\section{Hindawi}

Submit your manuscripts at

http://www.hindawi.com
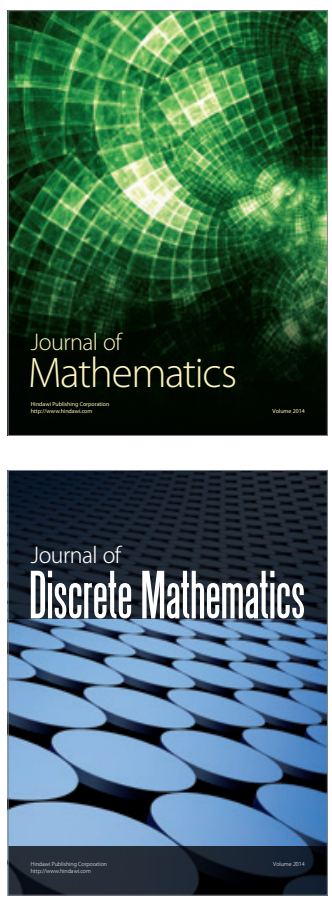

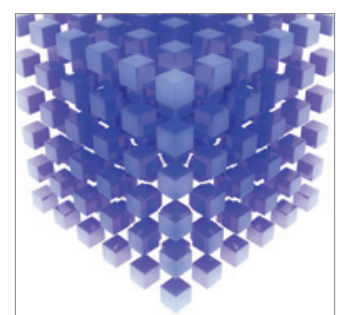

Mathematical Problems in Engineering
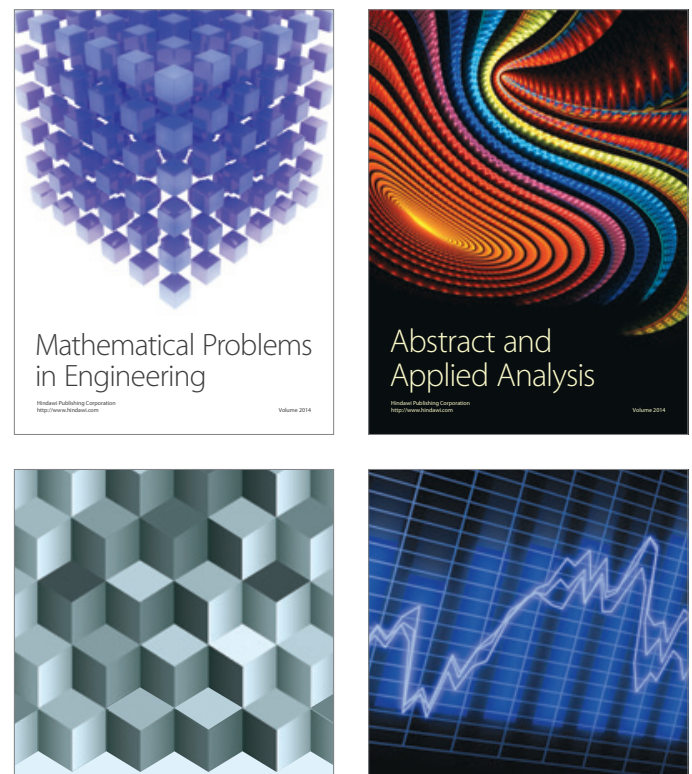

Journal of

Function Spaces

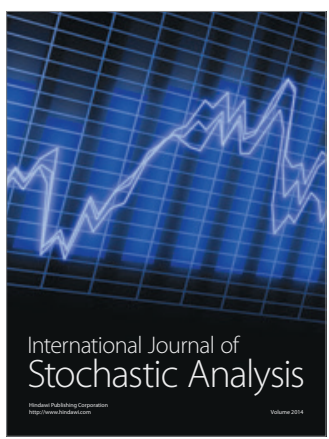

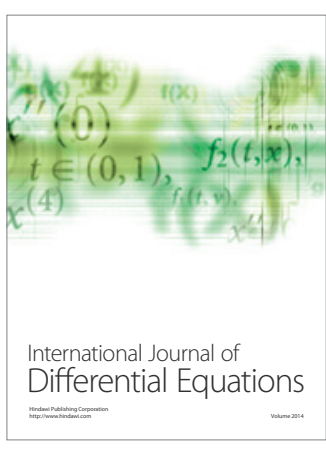
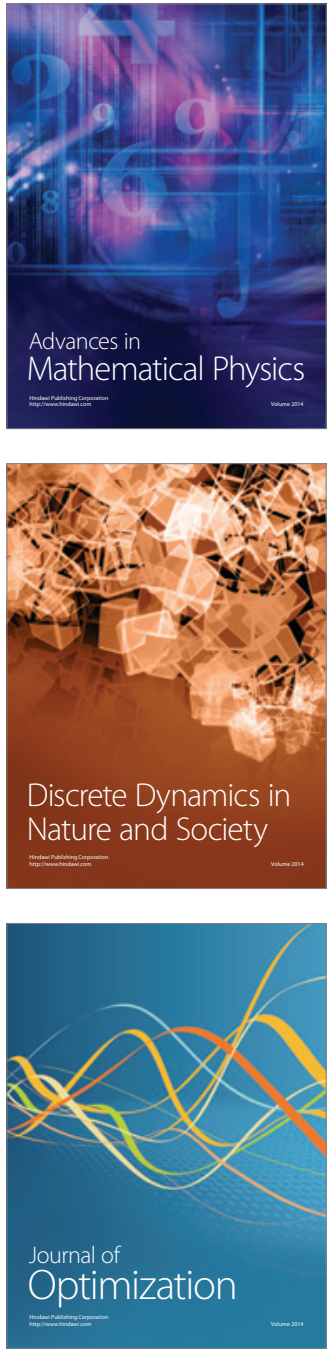